\title{
Nonlinear Resonance Responses of Electromechanical Integrated Magnetic Gear System
}

\author{
Xiu-hong Hao, Hong-qian Zhu, and Deng Pan \\ School of Mechanical Engineering, Yanshan University, Qinhuangdao, China \\ Correspondence should be addressed to Deng Pan; pandeng1896@sina.com
}

Received 28 September 2017; Revised 28 January 2018; Accepted 6 February 2018; Published 14 March 2018

Academic Editor: Tai Thai

Copyright (C) 2018 Xiu-hong Hao et al. This is an open access article distributed under the Creative Commons Attribution License, which permits unrestricted use, distribution, and reproduction in any medium, provided the original work is properly cited.

\begin{abstract}
Nonlinear differential equations for an electromechanical integrated magnetic gear (EIMG) system are developed by considering the nonlinearity in the magnetic force of the system components. Expressions for the main resonances and superharmonic resonances are obtained for output wave frequencies close to the natural frequency and half the natural frequency of the derived EIMG system. The response laws are discussed in detail. The magnetic coupling stiffness among the components is found to exhibit distinct nonlinearity, leading to strong main resonances and superharmonic resonances. Smaller values of the magnetic coupling stiffness and damping result in larger response amplitudes and transient responses that slowly decay to zero. When the main resonances and superharmonic resonances occur, the dominant frequency of the response is the natural frequency of the derived EIMG system, and the amplitudes of different components of the resonance display large differences.
\end{abstract}

\section{Introduction}

Magnetic drives, and especially magnetic gear boxes, are extensively used in the medical, food, and chemical industries. These systems have the advantages of being contactless, wear-free, and lubrication-free, with low levels of vibration and noise, and built-in overload protection [1]. The fieldmodulated magnetic gear (FMMG) has a coaxial topology, which significantly improves the permanent magnet (PM) utilization rate and can generate much higher torque and torque density than traditional magnetic gears that use a parallel shaft topology [2]. Therefore, FMMGs are widely used in the vehicle, wind, marine, and aerospace fields [3-5]. Various high-performance FMMG-based systems have been proposed, such as axial-flux magnetic gears, linear magnetic gears, and intersecting axes magnetic gears [6-8]. Various direct current PM rotors and alternating current PM motors have been developed for use in electric vehicles and wind power systems $[9,10]$. Together, these innovations have significantly promoted the development of magnetic gears.

Electrical and control techniques are widely used in mechanical engineering, resulting in the development of many cutting-edge composite drives such as the electromagnetic harmonic drive and electromechanical integrated toroidal drive. One of the authors has previously presented a drive system known as the electromechanical integrated magnetic gear (EIMG) [11]. This integrates power and control systems into FMMG. Thus, EIMG possesses the advantages of FMMG alongside a compact structure with torque and speed control. Compared with FMMG, EIMG can generate higher torque at lower speeds. EIMG can transmit torque by magnetic field coupling, unlike mechanical gears, which require direct contact to do so. The magnetic coupling stiffness of EIMG is much lower than the contact stiffness of mechanical gears $[12,13]$, and EIMG has different dynamic characteristics compared with mechanical gear systems. Because EIMG system has a lower magnetic coupling stiffness, its natural frequencies are much lower than those of mechanical gear systems. As a result, low-frequency resonance can easily occur. When such resonance occurs, the amplitudes of the EIMG system's components slowly attenuate, degrading the dynamics of the system. To overcome this problem, Frank et al. added damper windings to synchronous generators to suppress the oscillations caused by transients [14]. Montague et al. used servo control in magnetic gears to overcome the disadvantages of low stiffness [15]. 


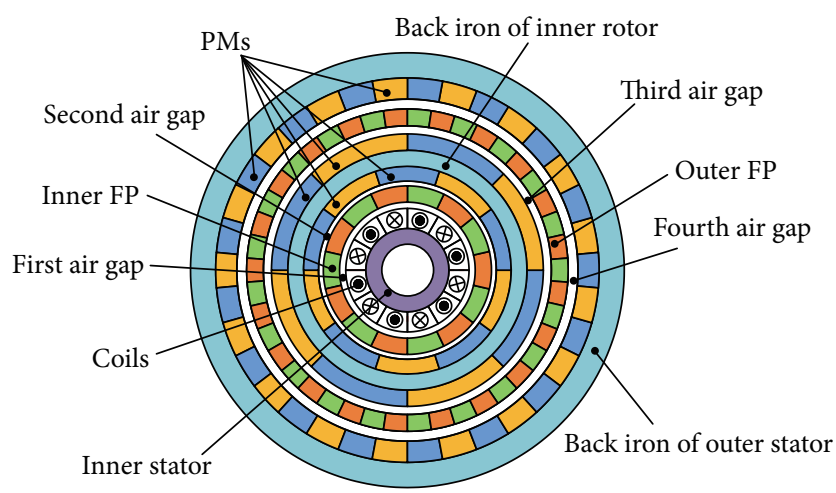

FIGURE 1: Topology of electromechanical integrated magnetic gear.

As EIMG is a relatively new type of magnetic gear, its dynamics should be thoroughly studied to control the behavior and improve the service performance of such a system. This study develops a dynamic model and differential equations for EIMG system by considering the nonlinearity of the magnetic coupling stiffness. The main resonances and superharmonic resonances produced by a torque wave are discussed, and these provide the foundation for the parameter optimization and performance improvement of the EIMG system.

\section{Nonlinear Dynamic Model of EIMG}

Figure 1 shows the inner rotor, inner and outer stator, and inner and outer ferromagnetic pole-pieces (FP) that comprise an EIMG system. All components are concentric, and the EIMG has four air gaps. Windings made of insulated wire are mounted in the dew-drop slot of the inner stator. PMs are positioned uniformly on the inner surface of the outer stator and the inner and outer surfaces of the inner rotor. The inner and outer FP comprise permeable and nonpermeable magnetic materials layered at regular intervals. FP modulate the magnetic fields in the two air gaps next to them, thus ensuring that the number of pole pairs of PMs on the inner rotor and outer stator agrees with the number of pole pairs of the space harmonic flux density of the air gaps.

A three-phase alternating current is supplied to the inner stator coils to generate a rotary magnetic field with a main harmonic of $p_{1}$. After being modulated by the inner FP, the main harmonic $p_{2}$ of the magnetic field in the second air gap is consistent with the number of pole pairs of PMs on the inner surface of the inner rotor. Therefore, the inner rotor can be actuated, thereby realizing the first drive. When the inner rotor runs, a magnetic field with a main harmonic of $p_{3}$ in the third air gap is generated and modulated by the outer FP. As a result, the main harmonic $p_{4}$ of the magnetic field in the fourth air gap becomes equal to the number of pole pairs of PMs on the outer stator. Then, the second drive is achieved.

To realize equal pole coupling among the EIMG components, $p_{1}, p_{2}, p_{3}$, and $p_{4}$ have the following constraint relations:

$$
\begin{aligned}
& p_{12}=p_{1}+p_{2}, \\
& p_{34}=p_{3}+p_{4},
\end{aligned}
$$

where $p_{1}$ is the number of pole pairs of the three-phase alternating current and $p_{4}, p_{2}$, and $p_{3}$ are the number of pole pairs of PMs on the stator and the inner and outer surfaces of the inner rotor, respectively.

The magnetic coupling torques among the components are calculated as follows:

$$
\begin{aligned}
& T_{1}=T_{c 1} \sin \left(p_{1} \theta_{12}+\theta_{10}\right), \\
& T_{3}=T_{c 3} \sin \left(p_{2} \theta_{23}+\theta_{10}\right)=T_{c 3} \sin \left(p_{3} \theta_{34}+\theta_{20}\right), \\
& T_{5}=T_{c 5} \sin \left(p_{4} \theta_{45}+\theta_{20}\right),
\end{aligned}
$$

where $T_{1}, T_{3}$, and $T_{5}$ are the torques on the inner stator, inner rotor, and outer stator, respectively; $T_{c 1}, T_{c 3}$, and $T_{c 5}$ are the maximum torques on the inner stator, inner rotor, and outer stator, respectively; $\theta_{10}$ and $\theta_{20}$ are the relative rotation angles between the inner stator and inner rotor and between the inner rotor and outer stator, respectively; and $\theta_{12}, \theta_{23}, \theta_{34}$, and $\theta_{45}$ are the relative torsional displacements between the inner stator and inner FP, inner FP and inner rotor, inner rotor and outer FP, and outer FP and outer stator, respectively.

Figure 2 shows the dynamic model of the EIMG system. This model contains four subsystems: inner stator/inner FP subsystem, inner FP/inner rotor subsystem, inner rotor/outer FP subsystem, and outer FP/outer stator subsystem. Because the magnetic coupling stiffness is much lower than the supporting stiffness [12], only torsional displacements are considered, and transverse vibration displacements are assumed to be negligible.

The tangential magnetic coupling forces among the components are calculated as follows:

$$
\begin{aligned}
& F_{\text {sI }}=\frac{T_{1}}{R_{1}}=\frac{T_{c 1} \sin \left[p_{1}\left(\theta_{1}-\theta_{2}\right)+\theta_{10}\right]}{R_{1}}, \\
& F_{\text {II }}=\frac{T_{3}}{R_{3}}=\frac{T_{c 3} \sin \left[p_{2}\left(\theta_{2}-\theta_{3}\right)+\theta_{10}\right]}{R_{3}}, \\
& F_{\text {Io }}=\frac{T_{3}}{R_{3}}=\frac{T_{c 3} \sin \left[p_{3}\left(\theta_{3}-\theta_{4}\right)+\theta_{20}\right]}{R_{3}}, \\
& F_{\text {oo }}=\frac{T_{5}}{R_{5}}=\frac{T_{c 5} \sin \left[p_{4}\left(\theta_{4}-\theta_{5}\right)+\theta_{20}\right]}{R_{5}},
\end{aligned}
$$

where $F_{\text {sI }}, F_{\text {II }}, F_{\text {Io }}$, and $F_{\text {oo }}$ are the tangential magnetic coupling forces between the inner stator and inner FP, inner FP and inner rotor, inner rotor and outer FP, and outer FP and outer stator, respectively; $R_{1}, R_{3}$, and $R_{5}$ are the equivalent radius of gyration of the inner stator, inner rotor, and outer stator, respectively; and $\theta_{k}(k=1,2,3,4,5)$ is the torsional angular displacement of the inner stator, inner FP, inner rotor, outer FP, and outer stator, respectively.

For convenience, the torsional angular displacements are all replaced by their corresponding torsional displacements as

$$
\mathbf{x}=\left[\begin{array}{lllll}
x_{1} & x_{2} & x_{3} & x_{4} & x_{5}
\end{array}\right]^{T},
$$

where $x_{k}(k=1,2,3,4,5)$ is the torsional displacement of the inner stator, inner FP, inner rotor, outer FP, and outer stator, respectively, and $x_{k}=R_{k} \theta_{k}$. 


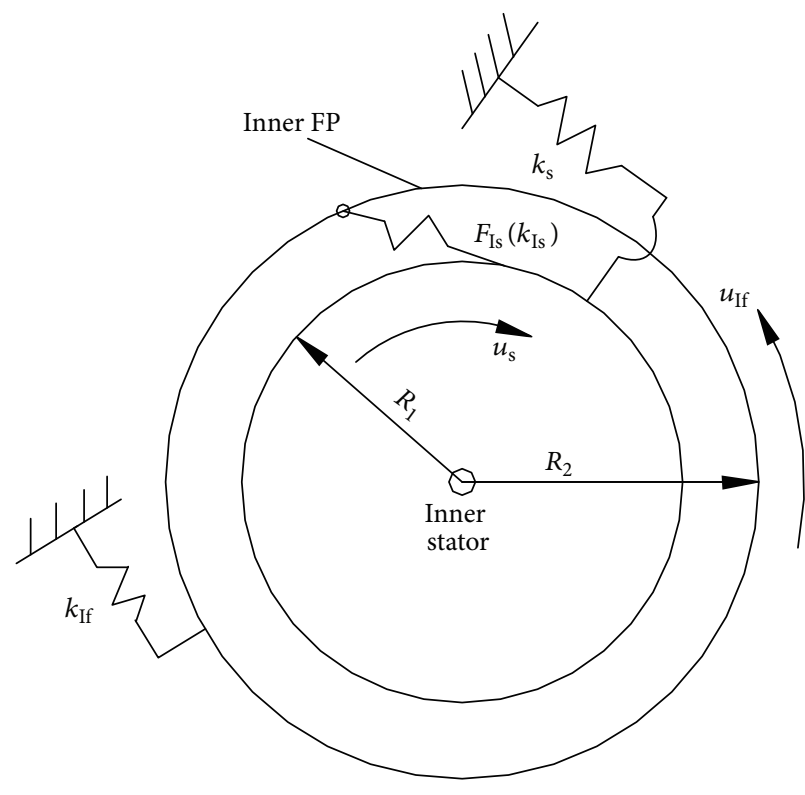

(a) Inner stator/inner FP subsystem

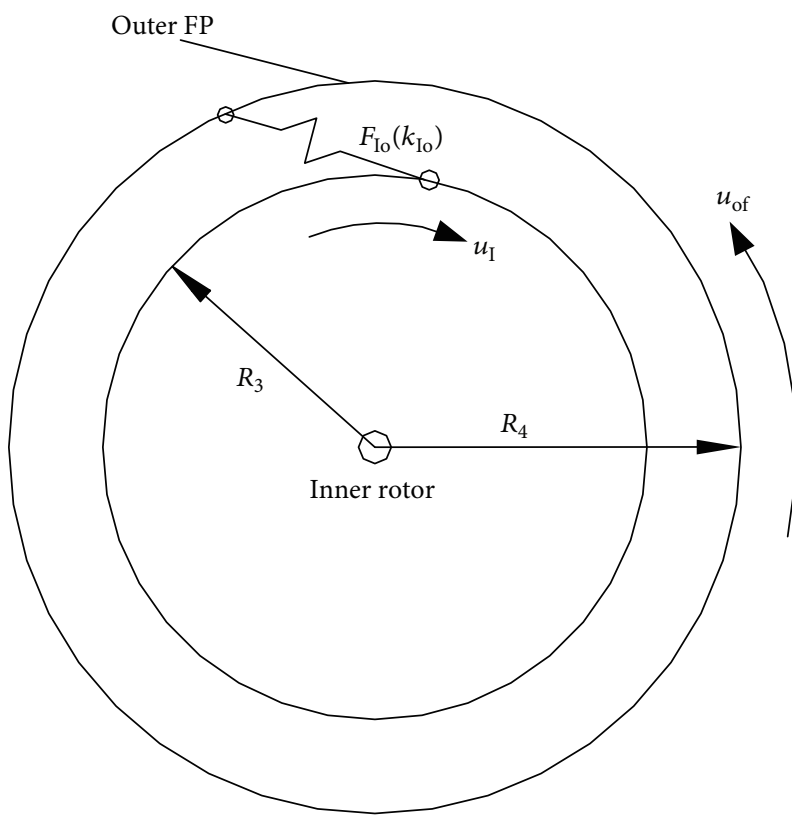

(c) Inner rotor/outer FP subsystem

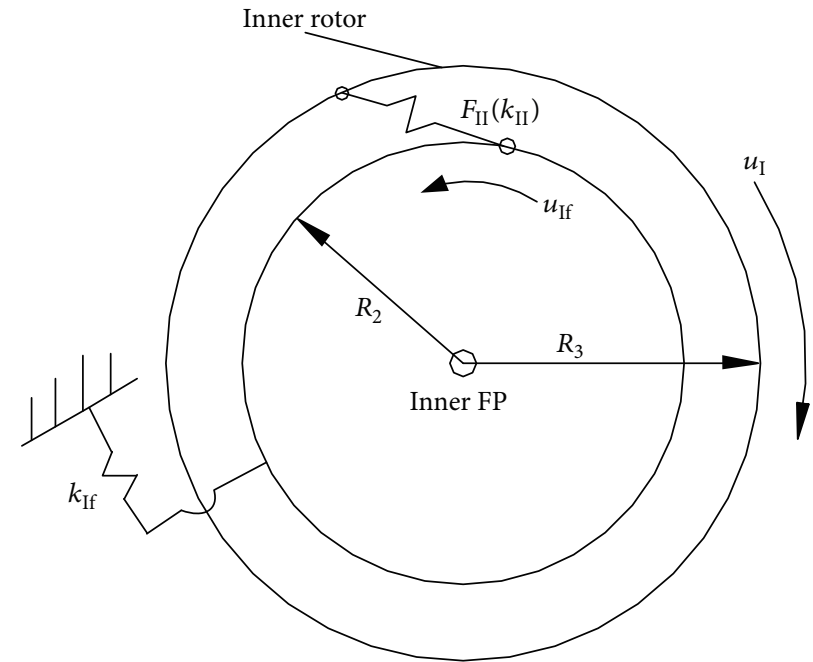

(b) Inner FP/inner rotor subsystem

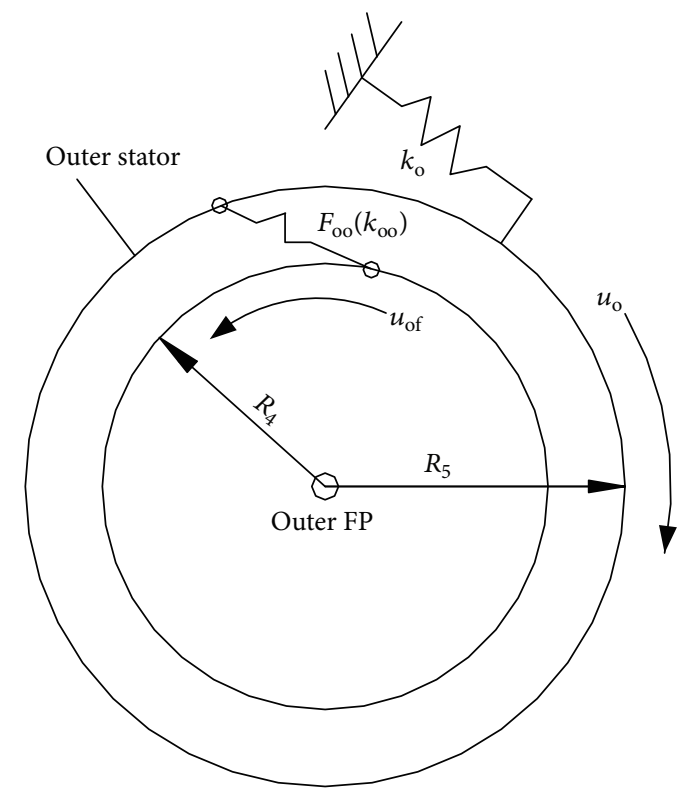

(d) Outer FP/outer stator subsystem

Figure 2: Nonlinear dynamic model of electromechanical integrated magnetic gear.

The magnetic coupling forces among the components are functions of the relative torsional displacements. When $t=0$, namely, $x_{i}=0$, the magnetic coupling forces are expanded using the Taylor series as

$$
\begin{aligned}
& F_{\mathrm{Is}}=h_{1}+a_{1} x_{1}+b_{1} x_{2}+c_{1} x_{1}^{2}+d_{1} x_{2}^{2}+e_{1} x_{1} x_{2}, \\
& F_{\mathrm{II}}=h_{2}+a_{2} x_{2}+b_{2} x_{3}+c_{2} x_{3}^{2}+d_{2} x_{2}^{2}+e_{2} x_{2} x_{3}, \\
& F_{\mathrm{Io}}=h_{3}+a_{3} x_{3}+b_{3} x_{4}+c_{3} x_{3}^{2}+d_{3} x_{4}^{2}+e_{3} x_{3} x_{4}, \\
& F_{\mathrm{oo}}=h_{4}+a_{4} x_{4}+b_{4} x_{5}+c_{4} x_{5}^{2}+d_{4} x_{4}^{2}+e_{4} x_{4} x_{5},
\end{aligned}
$$

where $h_{1}=T_{\mathrm{cs}} \sin \theta_{10} / R_{1}, a_{1}=T_{\mathrm{cs}} p_{1} \cos \theta_{10} / R_{1}^{3}, b_{1}$ $=-T_{\mathrm{cs}} p_{1} \cos ^{2} \theta_{10} / R_{1} R_{2}^{2}, c_{1}=T_{\mathrm{cs}} p_{1}^{2} \cos ^{2} \theta_{10} / 2 R_{1}^{5}, d_{1}=$ $T_{\mathrm{cs}} p_{1}^{2} \cos ^{2} \theta_{10} / 2 R_{1} R_{2}^{4}$, and $e_{1}=-T_{\mathrm{cs}} p_{1}^{2} \cos ^{2} \theta_{10} / R_{1}^{3} R_{2}^{2} ; h_{2}=$ $T_{\mathrm{cI}} \sin \theta_{10} / R_{3}, a_{2}=T_{\mathrm{cI}} p_{2} \cos \theta_{10} / R_{3} R_{2}^{2}, b_{2}=-T_{\mathrm{cI}} p_{2} \cos \theta_{10} /$ $R_{3}^{3}, c_{2}=T_{\mathrm{cl}} p_{2}^{2} \cos ^{2} \theta_{10} / 2 R_{3} R_{2}^{4}, d_{2}=T_{\mathrm{cl}} p_{2}^{2} \cos ^{2} \theta_{10} / 2 R_{3}^{5}$, and $e_{2}=-T_{\mathrm{cI}} p_{2}^{2} \cos ^{2} \theta_{10} / R_{3}^{3} R_{2}^{2} ; h_{3}=T_{\mathrm{cI}} \sin \theta_{20} / R_{3}, a_{3}=$ $T_{\mathrm{cl}} p_{3} \cos \theta_{20} / R_{3}^{3}, b_{3}=-T_{\mathrm{cI}} p_{3} \cos \theta_{20} / R_{3} R_{4}^{2}, c_{3}=T_{\mathrm{cI}} p_{3}^{2} \cos ^{2} \theta_{20} /$ $2 R_{3}^{5}, d_{3}=T_{\mathrm{cI}} p_{3}^{2} \cos ^{2} \theta_{20} / 2 R_{3} R_{4}^{4}$, and $e_{3}=T_{\mathrm{cI}} p_{3}^{2} \cos ^{2} \theta_{20} /$ $R_{3}^{3} R_{4}^{2}$; and $h_{4}=T_{\mathrm{co}} \sin \theta_{20} / R_{5}, a_{4}=T_{\mathrm{co}} p_{4} \cos \theta_{20} / R_{4}^{2} R_{5}$, $b_{4}=-T_{\mathrm{co}} p_{4} \cos \theta_{20} / R_{5}^{3}, c_{4}=T_{\mathrm{co}} p_{4}^{2} \cos ^{2} \theta_{20} / 2 R_{5}^{3} R_{4}^{2}, d_{4}=$ $T_{\text {co }} p_{4}^{2} \cos ^{2} \theta_{20} / 2 R_{5}^{5}$, and $e_{4}=-T_{\text {co }} p_{4}^{2} \cos ^{2} \theta_{20} / R_{4}^{2} R_{5}^{3}$. 
The magnetic coupling forces among the components are nonlinear functions of the relative torsional displacements. The constant components of the Taylor series expansions of the magnetic coupling forces will lead to relative static displacements rather than dynamic displacements. Therefore, $h_{i}$ is neglected. Based on Newton's second law of motion, the nonlinear dynamic differential equations of an EIMG system can be expressed as follows:

$$
\begin{aligned}
& M_{1} \ddot{x}_{1}+c_{\mathrm{s}} \dot{x}_{1}+a_{1} x_{1}+b_{1} x_{2}+c_{1} x_{1}^{2}+d_{1} x_{2}^{2}+e_{1} x_{1} x_{2} \\
& \quad+k_{\mathrm{s}} x_{1}=0, \\
& M_{2} \ddot{x}_{2}+c_{\mathrm{If}} \dot{x}_{2}-\left(a_{1} x_{1}+b_{1} x_{2}+c_{1} x_{1}^{2}+d_{1} x_{2}^{2}+e_{1} x_{1} x_{2}\right) \\
& \quad+\left(a_{2} x_{2}+b_{2} x_{3}+c_{2} x_{3}^{2}+d_{2} x_{2}^{2}+e_{2} x_{2} x_{3}\right)+k_{\mathrm{If}} x_{2} \\
& \quad=0, \\
& M_{3} \ddot{x}_{3}+c_{\mathrm{I}} \dot{x}_{3}-\left(a_{2} x_{2}+b_{2} x_{3}+c_{2} x_{3}^{2}+d_{2} x_{2}^{2}+e_{2} x_{2} x_{3}\right) \\
& \quad+\left(a_{3} x_{3}+b_{3} x_{4}+c_{3} x_{3}^{2}+d_{3} x_{4}^{2}+e_{3} x_{3} x_{4}\right)=0, \\
& M_{4} \ddot{x}_{4}+c_{\mathrm{of}} \dot{x}_{4}-\left(a_{3} x_{3}+b_{3} x_{4}+c_{3} x_{3}^{2}+d_{3} x_{4}^{2}+e_{3} x_{3} x_{4}\right) \\
& \quad+\left(a_{4} x_{4}+b_{4} x_{5}+c_{4} x_{5}^{2}+d_{4} x_{4}^{2}+e_{4} x_{4} x_{5}\right) \\
& \quad=\frac{\Delta T \cos \omega_{e} t}{R_{4}}, \\
& \quad+k_{\mathrm{o}} x_{5}=0, \\
& M_{5} \ddot{x}_{5}+c_{\mathrm{o}} \dot{x}_{5}-\left(a_{4} x_{4}+b_{4} x_{5}+c_{4} x_{5}^{2}+d_{4} x_{4}^{2}+e_{4} x_{4} x_{5}\right) \\
& \quad
\end{aligned}
$$

where $m_{1}$ and $m_{2}$ are the masses of the inner stator and inner FP, respectively; $M_{1}\left(M_{1}=J_{1} / R_{1}^{2}\right)$ and $M_{2}\left(M_{2}=J_{2} / R_{2}^{2}\right)$ are the equivalent masses of the inner stator and inner FP along their torsional vibration direction, respectively; $J_{1}\left(J_{1}=\right.$ $\left.m_{1} \cdot R_{1}^{2} / 2\right)$ and $J_{2}\left(J_{2}=m_{2} \cdot R_{2}^{2} / 2\right)$ are the mass moments of inertia of the inner stator and inner FP, respectively; $m_{3}$ and $M_{3}\left(M_{3}=J_{3} / R_{3}^{2}\right)$ are the mass and equivalent mass of the inner rotor, respectively; $J_{3}\left(J_{3}=m_{3} \cdot R_{3}^{2} / 2\right)$ is the mass moment of inertia of the inner rotor; $m_{4}$ and $M_{4}\left(M_{4}=\right.$ $\left.J_{4} / R_{4}^{2}\right)$ are the mass and equivalent mass of the outer FP, respectively; $J_{4}\left(J_{4}=m_{4} \cdot R_{4}^{2} / 2\right)$ is the mass moment of inertia of the outer FP; $m_{5}$ and $M_{5}\left(M_{5}=J_{5} / R_{5}^{2}\right)$ are the mass and equivalent mass of the outer stator, respectively; $J_{5}$ is the mass moment of inertia of the outer stator; $k_{\mathrm{s}}, k_{\mathrm{If}}$, and $k_{\mathrm{o}}$ are the torsional support stiffness values of the inner stator, inner FP, and outer stator, respectively; $c_{\mathrm{s}}, c_{\mathrm{If}}, c_{\mathrm{I}}, c_{\mathrm{of}}$, and $c_{\mathrm{o}}$ are the damping coefficients of the inner stator, inner FP, inner rotor, outer FP, and outer stator, respectively $\left(c_{\mathrm{s}}=\zeta_{\mathrm{s}} \sqrt{2 m_{1} a_{1} k_{\mathrm{s}} /\left(a_{1}+k_{\mathrm{s}}\right)}, c_{\mathrm{If}}=\zeta_{\mathrm{If}} \sqrt{2 m_{2} a_{1} a_{2} k_{\mathrm{If}} /\left(a_{1}+k_{\mathrm{If}}+a_{2}\right)}\right.$, $c_{\mathrm{I}}=\zeta_{\mathrm{I}} \sqrt{2 m_{3} a_{2} a_{3} /\left(a_{2}+a_{3}\right)}, c_{\text {of }}=\zeta_{\text {of }} \sqrt{2 m_{4} a_{3} a_{4} /\left(a_{3}+a_{4}\right)}$, and $c_{\mathrm{o}}=\zeta_{\mathrm{o}} \sqrt{2 m_{5} a_{4} k_{\mathrm{o}} /\left(a_{4}+k_{\mathrm{o}}\right)}$, where $\zeta_{\mathrm{s}}, \zeta_{\text {If }}, \zeta_{\mathrm{I}}, \zeta_{\mathrm{of}}$, and $\zeta_{\mathrm{o}}$ are the damping ratios of the inner stator, inner FP, inner rotor, outer FP, and outer stator, resp.).

Equation (6) can be rewritten in matrix form as

$$
\mathbf{M} \ddot{\mathbf{x}}+\mathbf{c} \dot{\mathbf{x}}+\mathbf{k x}=\mathbf{F}+\Delta \mathbf{F} .
$$

The mass matrix $\mathbf{M}$, displacement vector $\mathbf{x}$, damping matrix c, stiffness matrix $\mathbf{k}$, load vector $\mathbf{F}$, and equivalent load vector $\Delta \mathbf{F}$ have the following forms:

$$
\begin{aligned}
& \left.\mathbf{M}=\operatorname{diag}\left[\begin{array}{lllll}
M_{1} & M_{2} & M_{3} & M_{4} & M_{5}
\end{array}\right)\right] \\
& \mathbf{x}_{0}=\left[\begin{array}{lllll}
x_{1} & x_{2} & x_{3} & x_{4} & x_{5}
\end{array}\right]^{T}, \\
& \left.\mathbf{c}=\operatorname{diag}\left[\begin{array}{lllll}
c_{\mathrm{s}} & c_{\mathrm{If}} & c_{\mathrm{I}} & c_{\mathrm{of}} & c_{\mathrm{o}}
\end{array}\right)\right], \\
& \mathbf{k}=\left[\begin{array}{ccccc}
a_{1}+k_{\mathrm{s}} & b_{1} & 0 & 0 & 0 \\
-a_{1} & a_{2}-b_{1}+k_{\mathrm{If}} & b_{2} & 0 & 0 \\
0 & -a_{2} & a_{3}-b_{2} & b_{3} & 0 \\
0 & 0 & -a_{3} & a_{4}-b_{3} & b_{4} \\
0 & 0 & 0 & -a_{4} & k_{\mathrm{o}}-b_{4}
\end{array}\right] \text {, } \\
& \mathbf{F}=\left[\begin{array}{lllll}
F_{1} & F_{1} & F_{1} & F_{1} & F_{1}
\end{array}\right]^{T}, \\
& \Delta \mathbf{F}=\left[\begin{array}{lllll}
0 & 0 & 0 & \frac{\Delta T \cos \omega_{e} t}{R_{4}} & 0
\end{array}\right]^{T} \\
& F_{1}=-\left(c_{1} x_{1}^{2}+d_{1} x_{2}^{2}+e_{1} x_{1} x_{2}\right) \\
& F_{2}=c_{1} x_{1}^{2}+d_{1} x_{2}^{2}+e_{1} x_{1} x_{2}-\left(c_{2} x_{3}^{2}+d_{2} x_{2}^{2}+e_{2} x_{2} x_{3}\right) \\
& F_{3}=c_{2} x_{3}^{2}+d_{2} x_{2}^{2}+e_{2} x_{2} x_{3}-\left(c_{3} x_{3}^{2}+d_{3} x_{4}^{2}+e_{3} x_{3} x_{4}\right) \\
& F_{4}=c_{3} x_{3}^{2}+d_{3} x_{4}^{2}+e_{3} x_{3} x_{4}-\left(c_{4} x_{5}^{2}+d_{4} x_{4}^{2}+e_{4} x_{4} x_{5}\right) \\
& F_{5}=c_{4} x_{5}^{2}+d_{4} x_{4}^{2}+e_{4} x_{4} x_{5}
\end{aligned}
$$

where $\Delta T$ is the amplitude of the torque fluctuation and $\omega_{e}$ is the excitation frequency of the torque wave.

Equation (7) can be normalized as

$$
\ddot{\mathbf{x}}_{\mathrm{N}}+\mathbf{c}_{\mathrm{N}} \dot{\mathbf{x}}_{\mathrm{N}}+\mathbf{k}_{\mathrm{N}} \mathbf{x}_{\mathrm{N}}=\mathbf{F}_{\mathrm{N}}
$$

where $\mathbf{x}_{\mathrm{N}}, \mathbf{k}_{\mathrm{N}}$, and $\mathbf{F}_{\mathrm{N}}$ are the normal displacement vector, normal stiffness matrix, and normal equivalent load vector, respectively; these are expressed as follows:

$$
\begin{aligned}
\mathbf{x}_{\mathrm{N}}= & {\left[\begin{array}{lllll}
x_{\mathrm{N} 1} & x_{\mathrm{N} 2} & x_{\mathrm{N} 3} & x_{\mathrm{N} 4} & x_{\mathrm{N} 5}
\end{array}\right]^{T}, } \\
\mathbf{k}_{\mathrm{N}}= & \operatorname{diag}\left[\begin{array}{lllll}
k_{\mathrm{N} 1} & k_{\mathrm{N} 2} & k_{\mathrm{N} 3} & k_{\mathrm{N} 4} & \left.k_{\mathrm{N} 5}\right)
\end{array}\right] \\
= & \operatorname{diag}\left[\begin{array}{lllll}
\omega_{1} & \omega_{2} & \omega_{3} & \omega_{4} & \omega_{5}
\end{array}\right], \\
\mathbf{F}_{\mathrm{N}}= & {\left[\begin{array}{lllll}
F_{\mathrm{N} 1} & F_{\mathrm{N} 2} & F_{\mathrm{N} 3} & F_{\mathrm{N} 4} & F_{\mathrm{N} 5}
\end{array}\right]^{T}, } \\
F_{\mathrm{N} k}= & \frac{A_{\mathrm{N} 4 k} \Delta T \cos \omega_{e} t}{R_{4}}+A_{\mathrm{N} 1 i} F_{1}+A_{\mathrm{N} 2 i} F_{2}+A_{\mathrm{N} 3 i} F_{3} \\
& +A_{\mathrm{N} 4 i} F_{4}+A_{\mathrm{N} 5 i} F_{5},
\end{aligned}
$$

where $\omega_{i}$ is the natural frequency of the linear system derived for the EIMG.

Because the damping matrix $\mathbf{c}$ is diagonal, the elements on the primary diagonal of the normal damping matrix $\mathbf{c}_{\mathrm{N}}$ are much larger than the other elements. Therefore, the normal 
damping matrix $\mathbf{c}_{\mathrm{N}}$ in (9) is simplified into the following diagonal matrix:

$$
\mathbf{c}_{\mathrm{N}}=\operatorname{diag}\left[\left(\begin{array}{lllll}
c_{\mathrm{N} 1} & c_{\mathrm{N} 2} & c_{\mathrm{N} 3} & c_{\mathrm{N} 4} & c_{\mathrm{N} 5}
\end{array}\right)\right]
$$

\section{Main Resonance}

A multiscale method is used to solve (9). To balance the effects of the damping forces and nonlinear elements and express them in the same perturbation equation, the following assumptions are made:

$$
\begin{aligned}
x_{\mathrm{N} k} & =x_{\mathrm{N} k 0}\left(T_{0}, T_{1}\right)+\varepsilon x_{\mathrm{N} k 1}\left(T_{0}, T_{1}\right)+\cdots, \\
\Delta T & =\varepsilon \Delta T^{\prime}, \\
c_{\mathrm{N} k} & =\varepsilon c_{\mathrm{N} k}^{\prime} .
\end{aligned}
$$

When the wave frequency of the output torque is close to the natural frequency of the outer FP rotational mode, the following assumption is introduced:

$$
\omega_{e}=\omega_{4}+\varepsilon \sigma_{1} .
$$

By substituting (12) and (13) into (9) and setting the power coefficients of the small parameters $\varepsilon$ on both sides of the equations to be equal, the following differential equations are obtained:

Zero power

$$
D_{0}^{2} x_{\mathrm{N} k 0}+\omega_{k}^{2} x_{\mathrm{N} k 0}=0
$$

First power

$$
\begin{aligned}
D_{0}^{2} x_{\mathrm{N} k 1}+\omega_{k}^{2} x_{\mathrm{N} k 1}= & -c_{\mathrm{N} 1}^{\prime} D_{0} x_{\mathrm{N} k 0}-2 D_{0} D_{1} x_{\mathrm{N} k 0} \\
& +F_{\mathrm{N} k 0},
\end{aligned}
$$

where

$$
\begin{aligned}
F_{\mathrm{N} k 0}= & \frac{A_{\mathrm{N} 4 k} \Delta T^{\prime} \cos \omega_{e} t}{R_{4}}+A_{\mathrm{N} 1 k} F_{10}+A_{\mathrm{N} 2 k} F_{20} \\
& +A_{\mathrm{N} 3 k} F_{30}+A_{\mathrm{N} 4 k} F_{40}+A_{\mathrm{N} 5 k} F_{50}, \\
F_{10}= & -\left(c_{1} x_{10}^{2}+d_{1} x_{20}^{2}+e_{1} x_{10} x_{20}\right), \\
F_{20}= & c_{1} x_{10}^{2}+d_{1} x_{20}^{2}+e_{1} x_{10} x_{20} \\
& -\left(c_{2} x_{30}^{2}+d_{2} x_{20}^{2}+e_{2} x_{20} x_{30}\right), \\
F_{30}= & c_{2} x_{30}^{2}+d_{2} x_{20}^{2}+e_{2} x_{20} x_{30} \\
& -\left(c_{3} x_{30}^{2}+d_{3} x_{40}^{2}+e_{3} x_{30} x_{40}\right), \\
F_{40}= & c_{3} x_{30}^{2}+d_{3} x_{40}^{2}+e_{3} x_{30} x_{40} \\
& -\left(c_{4} x_{50}^{2}+d_{4} x_{40}^{2}+e_{4} x_{40} x_{50}\right), \\
F_{50}= & c_{4} x_{50}^{2}+d_{4} x_{40}^{2}+e_{4} x_{40} x_{50} .
\end{aligned}
$$

Under the normal coordinate system, the general solution of (14) is expressed as

$$
x_{\mathrm{N} k 0}=A_{k}\left(T_{1}\right) e^{i \omega_{k} T_{0}}+c c .
$$

Under the regular coordinate system, the solutions of (14) are

$$
\begin{aligned}
x_{10}= & \alpha_{1} A_{1}\left(T_{1}\right) e^{i \omega_{1} T_{0}}+\alpha_{2} A_{2}\left(T_{1}\right) e^{i \omega_{2} T_{0}} \\
& +\alpha_{3} A_{3}\left(T_{1}\right) e^{i \omega_{3} T_{0}}+\alpha_{4} A_{4}\left(T_{1}\right) e^{i \omega_{4} T_{0}} \\
& +\alpha_{5} A_{5}\left(T_{1}\right) e^{i \omega_{5} T_{0}}+c c, \\
x_{20}= & \beta_{1} A_{1}\left(T_{1}\right) e^{i \omega_{1} T_{0}}+\beta_{2} A_{2}\left(T_{1}\right) e^{i \omega_{2} T_{0}} \\
& +\beta_{3} A_{3}\left(T_{1}\right) e^{i \omega_{3} T_{0}}+\beta_{4} A_{4}\left(T_{1}\right) e^{i \omega_{4} T_{0}} \\
& +\beta_{5} A_{5}\left(T_{1}\right) e^{i \omega_{5} T_{0}}+c c, \\
x_{30}= & \gamma_{1} A_{1}\left(T_{1}\right) e^{i \omega_{1} T_{0}}+\gamma_{2} A_{2}\left(T_{1}\right) e^{i \omega_{2} T_{0}} \\
& +\gamma_{3} A_{3}\left(T_{1}\right) e^{i \omega_{3} T_{0}}+\gamma_{4} A_{4}\left(T_{1}\right) e^{i \omega_{4} T_{0}} \\
& +\gamma_{5} A_{5}\left(T_{1}\right) e^{i \omega_{5} T_{0}}+c c, \\
= & \mu_{1} A_{1}\left(T_{1}\right) e^{i \omega_{1} T_{0}}+\mu_{2} A_{2}\left(T_{1}\right) e^{i \omega_{2} T_{0}} \\
& +\mu_{3} A_{3}\left(T_{1}\right) e^{i \omega_{3} T_{0}}+\mu_{4} A_{4}\left(T_{1}\right) e^{i \omega_{4} T_{0}} \\
& +\mu_{5} A_{5}\left(T_{1}\right) e^{i \omega_{5} T_{0}}+c c, \\
x_{40} & \eta_{1} A_{1}\left(T_{1}\right) e^{i \omega_{1} T_{0}}+\eta_{2} A_{2}\left(T_{1}\right) e^{i \omega_{2} T_{0}} \\
& +\eta_{3} A_{3}\left(T_{1}\right) e^{i \omega_{3} T_{0}}+\eta_{4} A_{4}\left(T_{1}\right) e^{i \omega_{5} T_{0} T_{0}}+c c, \\
x_{50} & +{ }_{1},
\end{aligned}
$$

where $\alpha_{1}=A_{\mathrm{N} 11}, \alpha_{2}=A_{\mathrm{N} 12}, \alpha_{3}=A_{\mathrm{N} 13}, \alpha_{4}=A_{\mathrm{N} 14}$, and $\alpha_{5}=A_{\mathrm{N} 15} ; \beta_{1}=A_{\mathrm{N} 21}, \beta_{2}=A_{\mathrm{N} 22}, \beta_{3}=A_{\mathrm{N} 23}, \beta_{4}=A_{\mathrm{N} 24}$, and $\beta_{5}=A_{\mathrm{N} 25} ; \gamma_{1}=A_{\mathrm{N} 31}, \gamma_{2}=A_{\mathrm{N} 32}, \gamma_{3}=A_{\mathrm{N} 33}, \gamma_{4}=$ $A_{\mathrm{N} 34}$, and $\gamma_{5}=A_{\mathrm{N} 35} ; \mu_{1}=A_{\mathrm{N} 41}, \mu_{2}=A_{\mathrm{N} 42}, \mu_{3}=A_{\mathrm{N} 43}$, $\mu_{4}=A_{\mathrm{N} 44}$, and $\mu_{5}=A_{\mathrm{N} 45}$; and $\eta_{1}=A_{\mathrm{N} 51}, \eta_{2}=A_{\mathrm{N} 52}, \eta_{3}=$ $A_{\mathrm{N} 53}, \eta_{4}=A_{\mathrm{N} 54}$, and $\eta_{5}=A_{\mathrm{N} 55}$.

Here, $A_{\mathrm{N} k j}$ denotes the element in the $k$ th row and $j$ th column of the normal modal matrix $\mathbf{A}_{\mathrm{N}}$ :

$$
\mathbf{A}_{\mathrm{N}}=\left[\begin{array}{lllll}
A_{\mathrm{N} 11} & A_{\mathrm{N} 12} & A_{\mathrm{N} 13} & A_{\mathrm{N} 14} & A_{\mathrm{N} 15} \\
A_{\mathrm{N} 21} & A_{\mathrm{N} 22} & A_{\mathrm{N} 23} & A_{\mathrm{N} 24} & A_{\mathrm{N} 25} \\
A_{\mathrm{N} 31} & A_{\mathrm{N} 32} & A_{\mathrm{N} 33} & A_{\mathrm{N} 34} & A_{\mathrm{N} 35} \\
A_{\mathrm{N} 41} & A_{\mathrm{N} 42} & A_{\mathrm{N} 42} & A_{\mathrm{N} 42} & A_{\mathrm{N} 42} \\
A_{\mathrm{N} 51} & A_{\mathrm{N} 52} & A_{\mathrm{N} 53} & A_{\mathrm{N} 54} & A_{\mathrm{N} 55}
\end{array}\right] .
$$

By substituting (18), (15) can be rewritten as

$$
\begin{aligned}
& D_{0}^{2} x_{\mathrm{N} k 1}+\omega_{k}^{2} x_{\mathrm{N} k 1} \\
& =-c_{\mathrm{N} 1}^{\prime} i \omega_{k} A_{k} e^{i \omega_{k} T_{0}}-2\left(A_{k}^{\prime} e^{i \omega_{k} T_{0}}+i \omega_{k} A_{k} e^{i \omega_{k} T_{0}}\right) \\
& \quad+\frac{A_{\mathrm{N} 4 k} \Delta T^{\prime} \cos \omega_{e} t}{R_{4}}
\end{aligned}
$$




$$
\begin{aligned}
& +\sum_{k=1}^{5} \sum_{j=1}^{5} G_{k j} A_{k}\left[A_{j} e^{i\left(\omega_{k}+\omega_{j}\right) T_{0}}+\bar{A}_{j} e^{i\left(\omega_{k}-\omega_{j}\right) T_{0}}\right] \\
& +c c
\end{aligned}
$$

where $c c$ is the complex conjugate of the right-hand-side expression in (20) and $G_{k j}$ is a constant associated with $\mathbf{A}_{\mathrm{N}}, c_{k}$, $d_{k}$, and $e_{k}$ that is expressed as follows:

$$
\begin{aligned}
G_{k j} & =-A_{\mathrm{N} 1 k}\left(c_{1} \alpha_{k} \alpha_{j}+d_{1} \beta_{k} \beta_{j}+e_{1} \alpha_{k} \beta_{j}\right) \\
& +A_{\mathrm{N} 2 k}\left[\left(c_{1} \alpha_{k} \alpha_{j}+d_{1} \beta_{k} \beta_{j}+e_{1} \alpha_{k} \beta_{j}\right)\right. \\
& \left.-\left(c_{2} \gamma_{k} \gamma_{j}+d_{2} \beta_{k} \beta_{j}+e_{2} \gamma_{k} \beta_{j}\right)\right] \\
& +A_{\mathrm{N} 3 k}\left[\left(c_{2} \gamma_{k} \gamma_{j}+d_{2} \beta_{k} \beta_{j}+e_{2} \gamma_{k} \beta_{j}\right)\right. \\
& \left.-\left(c_{3} \gamma_{k} \gamma_{j}+d_{3} \mu_{k} \mu_{j}+e_{3} \gamma_{k} \mu_{j}\right)\right] \\
& +A_{\mathrm{N} 4 k}\left[\left(c_{3} \gamma_{k} \gamma_{j}+d_{3} \mu_{k} \mu_{j}+e_{3} \gamma_{k} \mu_{j}\right)\right. \\
& \left.-\left(c_{4} \eta_{k} \eta_{j}+d_{4} \mu_{k} \mu_{j}+e_{4} \eta_{m} \mu_{j}\right)\right]+A_{\mathrm{N} 5 i}\left(c_{4} \eta_{k} \eta_{j}\right. \\
& \left.+d_{4} \mu_{k} \mu_{j}+e_{4} \eta_{k} \mu_{j}\right)
\end{aligned}
$$

There are multiple frequency components in the righthand-side expressions of (20), such as $\pm 2 \omega_{k}, \pm\left(\omega_{k} \pm \omega_{j}\right)$, and $\pm \omega_{e}$, where $m=1,2,3,4,5$ and $k \neq j$. When there is no internal resonance, the secular terms can be eliminated to obtain

$$
\begin{array}{r}
-c_{\mathrm{N} m}^{\prime} A_{m}-2 \dot{A}_{m}=0, \\
-c_{\mathrm{N} 4}^{\prime} i \omega_{4} A_{4}-2 i \omega_{4} \dot{A}_{4}+\frac{A_{\mathrm{N} 44} \Delta T^{\prime} e^{i \varepsilon \sigma_{1} t}}{R_{4}}=0,
\end{array}
$$

where $m=1,2,3$, and 5 .

Solutions of (22) are obtained as follows:

$$
\begin{aligned}
& A_{m}=E_{m} e^{-c_{\mathrm{N} k} t / 2}, \\
& A_{4}=E_{4} e^{-c_{\mathrm{N} 4} t / 2}-\frac{A_{\mathrm{N} 44} \Delta T^{\prime} e^{i\left(\varepsilon \sigma_{1} t+\theta_{1}\right)}}{R_{\mathrm{of}} \omega_{4} \sqrt{c_{\mathrm{N} 4}^{2}+4 \varepsilon^{2} \sigma_{1}^{2}}}
\end{aligned}
$$

where $E_{k}$ and $E_{4}$ are constants related to the output torque, $\varphi_{1}$ is a constant related to $\varepsilon$ and $\sigma, \cos \varphi_{1}=2 \varepsilon \sigma_{1} / \sqrt{c_{\mathrm{N} 4}^{\prime 2}+4 \varepsilon^{2} \sigma_{1}^{2}}$, and $\sin \varphi_{1}=c_{\mathrm{N} 4} / \sqrt{c_{\mathrm{N} 4}^{\prime 2}+4 \varepsilon^{2} \sigma_{1}^{2}}$.

Under the normal coordinate system, the zero-order approximate analytical solution of the EIMG system is expressed as

$$
\begin{aligned}
& x_{\mathrm{N} m 0}=E_{m} e^{-c_{\mathrm{N} m}^{\prime} t / 2} e^{i \omega_{m} T_{0}}+c c, \\
& x_{\mathrm{N} 40}=E_{4} e^{-c_{\mathrm{N} 4}^{\prime} t / 2} e^{i \omega_{4} T_{0}}-\frac{A_{\mathrm{N} 44} \Delta T^{\prime} e^{i\left(\omega_{4} t+\varepsilon \sigma_{1} t+\varphi_{1}\right)}}{R_{4} \omega_{4} \sqrt{c_{\mathrm{N} 4}^{2}+4 \varepsilon^{2} \sigma_{1}^{2}}}+c c .
\end{aligned}
$$

After eliminating the secular terms, the first-order approximate solutions are obtained by substituting (23) and (24) into (20):

$$
\begin{aligned}
& x_{\mathrm{N} m 1}=\frac{A_{\mathrm{N} 44} \Delta T^{\prime} e^{j \omega_{e} t}}{R_{4}\left(\omega_{m}^{2}-\omega_{e}^{2}\right)}+x_{\mathrm{N} 41} \text {, } \\
& x_{\mathrm{N} 41}=\sum_{m=1,2,3,5} \sum_{j=1,2,3,5} G_{m j} E_{m} E_{j} e^{-\left(c_{\mathrm{Nj}}^{\prime}+c_{\mathrm{N} m}^{\prime}\right) t / 2} \\
& \cdot \frac{e^{i\left(\omega_{m} \pm \omega_{j}\right) t}}{\omega_{m}^{2}-\left(\omega_{m} \pm \omega_{j}\right)^{2}} \\
& +\sum_{m=1,2,3,5} G_{m 4} E_{m}\left\{\frac{E_{4} e^{-\left(c_{\mathrm{N} 4}^{\prime}+c_{\mathrm{N} m}\right) t / 2} e^{i\left(\omega_{m} \pm \omega_{4}\right) t}}{\omega_{m}^{2}-\left(\omega_{m} \pm \omega_{4}\right)^{2}}\right. \\
& \left.-\frac{X_{4} E_{m} e^{-c_{\mathrm{N} m}^{\prime} t / 2} e^{i\left[\left(\omega_{m}+\omega_{4}\right) t \pm\left(\varepsilon \sigma_{1} t+\varphi_{1}\right)\right]}}{\omega_{m}^{2}-\left(\omega_{m}+\omega_{4}+\varepsilon \sigma_{1}\right)^{2}}\right\} \\
& +\sum_{j=1,2,3,5} G_{4 j} E_{j}\left\{\frac{E_{4} e^{-\left(c_{\mathrm{N} 4}^{\prime}+c_{\mathrm{N} j}^{\prime}\right) t / 2} e^{i\left(\omega_{4} \pm \omega_{j}\right) T_{0}}}{\omega_{m}^{2}-\left(\omega_{4} \pm \omega_{j}\right)^{2}}\right. \\
& \left.-\frac{X e^{-c_{\mathrm{N} j}^{\prime} t / 2} e^{i\left[\left(\omega_{4}+\omega_{j}\right) t \pm\left(\varepsilon \sigma_{1} t+\varphi_{1}\right)\right]}}{\omega_{m}^{2}-\left(\omega_{4}+\omega_{j}+\varepsilon \sigma_{1}\right)^{2}}\right\}+\left\{\frac{E_{4}^{2} e^{-c_{\mathrm{N} 4}^{\prime} t} e^{2 i \omega_{4} t}}{\omega_{4}^{2}-\left(2 \omega_{4}\right)^{2}}\right. \\
& \left.+\frac{X_{4} E_{4} e^{-c_{\mathrm{N} 4}^{\prime} t / 2} e^{i\left[\left(\omega_{m}+\omega_{4}\right) t+\varepsilon \sigma_{1} t+\varphi_{1}\right]}}{\omega_{4}^{2}-\left(2 \omega_{4}+\varepsilon \sigma_{1}\right)^{2}}\right\} \\
& -\left(\frac{X_{4} E_{4} e^{-c_{\mathrm{N} 4}^{\prime} t / 2} e^{i\left(2 \omega_{4} t+\varepsilon \sigma_{1} t+\varphi_{1}\right)}}{\omega_{4}^{2}-\left(2 \omega_{4}+\varepsilon \sigma_{1}\right)^{2}}\right. \\
& \left.-\frac{X_{4}^{2} e^{i\left(2 \omega_{4} t+2 \varepsilon \sigma_{1} t+2 \varphi_{1}\right)}}{\omega_{4}^{2}-\left(2 \omega_{4}+2 \varepsilon \sigma_{1}\right)^{2}}\right)+c c,
\end{aligned}
$$

where

$$
\begin{aligned}
X_{4} & =\frac{A_{\mathrm{N} 44} \Delta T^{\prime}}{R_{4} \omega_{4} \sqrt{c_{\mathrm{N} 4}^{\prime 2}+4 \varepsilon^{2} \sigma_{1}^{2}}} ; \\
G_{m j} & =-A_{\mathrm{N} 1 i}\left(c_{1} \alpha_{m} \alpha_{j}+d_{1} \beta_{m} \beta_{j}+e_{1} \alpha_{m} \beta_{j}\right) \\
& +A_{\mathrm{N} 2 i}\left[\left(c_{1} \alpha_{m} \alpha_{j}+d_{1} \beta_{m} \beta_{j}+e_{1} \alpha_{m} \beta_{j}\right)\right. \\
& \left.-\left(c_{2} \gamma_{m} \gamma_{j}+d_{2} \beta_{m} \beta_{j}+e_{2} \gamma_{m} \beta_{j}\right)\right] \\
& +A_{\mathrm{N} 3 i}\left[\left(c_{2} \gamma_{m} \gamma_{j}+d_{2} \beta_{m} \beta_{j}+e_{2} \gamma_{m} \beta_{j}\right)\right. \\
& \left.-\left(c_{3} \gamma_{m} \gamma_{j}+d_{3} \mu_{m} \mu_{j}+e_{3} \gamma_{m} \mu_{j}\right)\right]+A_{\mathrm{N} 5 i}\left(c_{4} \eta_{m} \eta_{j}\right. \\
& \left.+d_{4} \mu_{m} \mu_{j}+e_{4} \eta_{m} \mu_{j}\right) \\
& +A_{\mathrm{N} 4 i}\left[\left(c_{3} \gamma_{m} \gamma_{j}+d_{3} \mu_{m} \mu_{j}+e_{3} \gamma_{m} \mu_{j}\right)\right. \\
& \left.-\left(c_{4} \eta_{m} \eta_{j}+d_{4} \mu_{m} \mu_{j}+e_{4} \eta_{m} \mu_{j}\right)\right] .
\end{aligned}
$$


$E_{m}$ will approach zero with increasing time because of the damping among components. The stable responses of the first-order approximate analytic solution of the EIMG system can be obtained by substituting (24) and (25) into (12):

$$
\begin{aligned}
& x_{\mathrm{N} m}=\frac{\varepsilon A_{\mathrm{N} 44} \Delta T^{\prime} e^{i \omega_{e} t}}{R_{4}\left(\omega_{m}^{2}-\omega_{e}^{2}\right)}+\frac{\varepsilon X_{4}^{2} e^{i\left(2 \omega_{4} t+2 \varepsilon \sigma_{1} t+2 \varphi_{1}\right)}}{\omega_{4}^{2}-\left(2 \omega_{4}+2 \varepsilon \sigma_{1}\right)^{2}}+c \mathcal{C}, \\
& x_{\mathrm{N} 4}=-\frac{A_{\mathrm{N} 44} \Delta T^{\prime} e^{i\left(\omega_{4} t+\varepsilon \sigma_{1} t+\theta_{1}\right)}}{R_{4} \omega_{4} \sqrt{c_{\mathrm{N} 4}^{\prime 2}+4 \varepsilon^{2} \sigma_{1}^{2}}}+\frac{\varepsilon X_{4}^{2} e^{i\left(2 \omega_{4} t+2 \varepsilon \sigma_{1} t+2 \varphi_{1}\right)}}{\omega_{4}^{2}-\left(2 \omega_{4}+2 \varepsilon \sigma_{1}\right)^{2}}
\end{aligned}
$$

$+c c$.

Based on (27), approximate analytic solutions of the EIMG system under the regular coordinate system are given by

$$
\mathbf{x}=\mathbf{A}_{\mathrm{N}} \mathbf{x}_{\mathrm{N}} .
$$

When the torque wave frequency approaches a certain frequency $\omega_{k}$ of the derived EIMG system, resonance will occur. In this situation, stable responses of the EIMG system under the normal coordinate system are calculated as

$$
\begin{aligned}
x_{\mathrm{N} j}= & \frac{\varepsilon A_{\mathrm{N} 4 j} \Delta T^{\prime} e^{i \omega_{e} t}}{R_{4}\left(\omega_{j}^{2}-\omega_{e}^{2}\right)}+\frac{\varepsilon X_{j}^{2} e^{i\left(2 \omega_{j} t+2 \varepsilon \sigma_{1} t+2 \varphi_{1}\right)}}{\omega_{j}^{2}-\left(2 \omega_{j}+2 \varepsilon \sigma_{1}\right)^{2}}+c c, \\
x_{\mathrm{N} k}= & -\frac{A_{\mathrm{N} 4 j} \Delta T^{\prime} e^{i\left(\omega_{j} t+\varepsilon \sigma_{1} t+\theta_{1}\right)}}{R_{4} \omega_{j} \sqrt{c_{\mathrm{N} j}^{\prime 2}+4 \varepsilon^{2} \sigma_{1}^{2}}}+\frac{\varepsilon X_{j}^{2} e^{i\left(2 \omega_{j} t+2 \varepsilon \sigma_{1} t+2 \varphi_{1}\right)}}{\omega_{j}^{2}-\left(2 \omega_{j}+2 \varepsilon \sigma_{1}\right)^{2}} \\
& +c c
\end{aligned}
$$

where $X_{k}=A_{\mathrm{N} 44} \Delta T^{\prime} / R_{4} \omega_{k} \sqrt{c_{\mathrm{N} k}^{\prime 2}+4 \varepsilon^{2} \sigma_{1}^{2}}$.

\section{Superharmonic Resonance}

When the wave frequency of the output torque is close to half of the natural frequency of the outer FP rotational mode, the following assumption is introduced:

$$
\begin{aligned}
& x_{\mathrm{N} j}=x_{\mathrm{N} j 0}\left(T_{0}, T_{1}\right)+\varepsilon x_{\mathrm{N} j 1}\left(T_{0}, T_{1}\right)+\cdots, \\
& 2 \omega_{e}=\omega_{4}+\varepsilon \sigma_{2}, \\
& c_{\mathrm{N} j}=\varepsilon c_{\mathrm{N} j}^{\prime} .
\end{aligned}
$$

By substituting (30) into (9) and setting the power coefficients of the small parameters $\varepsilon$ on both sides of the equation to be equal, the following differential equations are obtained:

\section{Zero power}

$$
D_{0}^{2} x_{\mathrm{N} j 0}+\omega_{j}^{2} x_{\mathrm{N} j 0}=F_{\mathrm{N} j 0}^{\prime}
$$

First power

$$
\begin{aligned}
& D_{0}^{2} x_{\mathrm{N} k 1}+\omega_{k}^{2} x_{\mathrm{N} k 1}=-c_{\mathrm{N} 1}^{\prime} i \omega_{k} A_{k} e^{i \omega_{k} T_{0}}-2\left(A_{k}^{\prime} e^{i \omega_{k} T_{0}}\right. \\
& \left.+i \omega_{k} A_{k} e^{i \omega_{k} T_{0}}\right)+L_{k} e^{2 j \omega_{e} T_{0}} \\
& +L_{k} \sum_{m=1}^{5} H_{k}\left(A_{m} e^{i\left(\omega_{m}+\omega_{e}\right) T_{0}}+\bar{A}_{m} e^{i\left(-\omega_{m}+\omega_{e}\right) T_{0}}\right) \\
& +\sum_{k=1}^{5} \sum_{j=1}^{5} G_{k j} A_{k}\left[A_{j} e^{i\left(\omega_{k}+\omega_{j}\right) T_{0}}+\bar{A}_{j} e^{i\left(\omega_{k}-\omega_{j}\right) T_{0}}\right]+c c,
\end{aligned}
$$

where

$$
\begin{aligned}
F_{\mathrm{N} j 0} & =\frac{A_{\mathrm{N} 4 j} \Delta T \cos \omega_{e} t}{R_{4}} \\
F_{\mathrm{N} j 0}^{\prime \prime} & =A_{\mathrm{N} 1 j} F_{10}+A_{\mathrm{N} 2 j} F_{20}+A_{\mathrm{N} 3 j} F_{30}+A_{\mathrm{N} 4 j} F_{40} \\
& +A_{\mathrm{N} 5 j} F_{50}, \\
H_{k} & =A_{\mathrm{N} 1 i}\left[c_{1} \alpha_{m}+d_{1} \beta_{m} \rho_{2}+e_{1}\left(\beta_{m} \rho_{1}+\alpha_{m} \rho_{2}\right)\right] \\
& +A_{\mathrm{N} 2 i}\left[c_{1} \alpha_{m}+d_{1} \beta_{m} \rho_{2}+e_{1}\left(\beta_{m} \rho_{1}+\alpha_{m} \rho_{2}\right)\right. \\
& \left.-c_{2} \gamma_{m} \rho_{3}-d_{2} \beta_{m} \rho_{2}-e_{2}\left(\beta_{m} \rho_{3}+\gamma_{m} \rho_{2}\right)\right] \\
& +A_{\mathrm{N} 3 i}\left[c_{2} \gamma_{m} \rho_{3}+d_{2} \beta_{m} \rho_{2}+e_{2}\left(\beta_{m} \rho_{3}+\gamma_{m} \rho_{2}\right)\right. \\
& \left.-c_{3} \gamma_{m} \rho_{3}-d_{3} \mu_{m} \rho_{4}-e_{3}\left(\gamma_{m} \rho_{4}+\mu_{m} \rho_{3}\right)\right] \\
& +A_{\mathrm{N} 4 i}\left[c_{3} \gamma_{m} \rho_{3}+d_{3} \mu_{m} \rho_{4}+e_{3}\left(\gamma_{m} \rho_{4}+\mu_{m} \rho_{3}\right)\right. \\
& \left.-c_{4} \gamma_{m} \rho_{5}-d_{4} \mu_{m} \rho_{4}-e_{4}\left(\eta_{m} \rho_{4}+\mu_{m} \rho_{5}\right)\right] \\
& +A_{\mathrm{N} 5 i}\left[c_{4} \gamma_{m} \rho_{5}+d_{4} \mu_{m} \rho_{4}+e_{4}\left(\eta_{m} \rho_{4}+\mu_{m} \rho_{5}\right)\right] \\
L_{k} & =A_{\mathrm{N} 1 i}\left(c_{1} \rho_{1}^{2}+d_{1} \rho_{2}^{2}+e_{1} \rho_{1} \rho_{2}\right)+A_{\mathrm{N} 2 i}\left[c_{1} \rho_{1}^{2}\right. \\
& \left.+d_{1} \rho_{2}^{2}+e_{1} \rho_{1} \rho_{2}-\left(c_{2} \rho_{3}^{2}+d_{2} \rho_{2}^{2}+e_{2} \rho_{2} \rho_{3}\right)\right] \\
& +A_{\mathrm{N} 3 i}\left[c_{2} \rho_{3}^{2}+d_{2} \rho_{2}^{2}+e_{2} \rho_{2} \rho_{3}\right. \\
& \left.-\left(c_{3} \rho_{3}^{2}+d_{3} \rho_{4}^{2}+e_{3} \rho_{3} \rho_{4}\right)\right]+A_{\mathrm{N} 4 i}\left[c_{3} \rho_{3}^{2}+d_{3} \rho_{4}^{2}\right. \\
& \left.+e_{3} \rho_{3} \rho_{4}-\left(c_{4} \rho_{5}^{2}+d_{4} \rho_{4}^{2}+e_{4} \rho_{4} \rho_{5}\right)\right]+A_{\mathrm{N} 5 i}\left(c_{4} \rho_{5}^{2}\right. \\
& \left.+d_{4} \rho_{4}^{2}+e_{4} \rho_{4} \rho_{5}\right) . \\
&
\end{aligned}
$$

Under the normal coordinate system, the general solution of (31) is expressed as

$$
x_{\mathrm{N} j 0}=A_{j}\left(T_{1}\right) e^{i \omega_{j} T_{0}}+B_{j} e^{i \omega_{e} T_{0}}+c c,
$$

where $B_{j}=A_{\mathrm{N} 4 j} \Delta T / R_{4}\left(\omega_{j}^{2}-\omega_{e}^{2}\right)$. 
Under the regular coordinate system, solutions of (31) are calculated as

$$
\begin{aligned}
& x_{10}=\alpha_{1} A_{1}\left(T_{1}\right) e^{i \omega_{1} T_{0}}+\alpha_{2} A_{2}\left(T_{1}\right) e^{i \omega_{2} T_{0}} \\
& +\alpha_{3} A_{3}\left(T_{1}\right) e^{i \omega_{3} T_{0}}+\alpha_{4} A_{4}\left(T_{1}\right) e^{i \omega_{4} T_{0}} \\
& +\alpha_{5} A_{5}\left(T_{1}\right) e^{i \omega_{5} T_{0}}+\rho_{1} e^{j \omega_{e} T_{0}}+c c, \\
& x_{20}=\beta_{1} A_{1}\left(T_{1}\right) e^{i \omega_{1} T_{0}}+\beta_{2} A_{2}\left(T_{1}\right) e^{i \omega_{2} T_{0}} \\
& +\beta_{3} A_{3}\left(T_{1}\right) e^{i \omega_{3} T_{0}}+\beta_{4} A_{4}\left(T_{1}\right) e^{i \omega_{4} T_{0}} \\
& +\beta_{5} A_{5}\left(T_{1}\right) e^{i \omega_{5} T_{0}}+\rho_{2} e^{j \omega_{e} T_{0}}+c c, \\
& x_{30}=\gamma_{1} A_{1}\left(T_{1}\right) e^{i \omega_{1} T_{0}}+\gamma_{2} A_{2}\left(T_{1}\right) e^{i \omega_{2} T_{0}} \\
& +\gamma_{3} A_{3}\left(T_{1}\right) e^{i \omega_{3} T_{0}}+\gamma_{4} A_{4}\left(T_{1}\right) e^{i \omega_{4} T_{0}} \\
& +\gamma_{5} A_{5}\left(T_{1}\right) e^{i \omega_{5} T_{0}}+\rho_{3} e^{j \omega_{e} T_{0}}+c c, \\
& x_{40}=\mu_{1} A_{1}\left(T_{1}\right) e^{i \omega_{1} T_{0}}+\mu_{2} A_{2}\left(T_{1}\right) e^{i \omega_{2} T_{0}} \\
& +\mu_{3} A_{3}\left(T_{1}\right) e^{i \omega_{3} T_{0}}+\mu_{4} A_{4}\left(T_{1}\right) e^{i \omega_{4} T_{0}} \\
& +\mu_{5} A_{5}\left(T_{1}\right) e^{i \omega_{5} T_{0}}+\rho_{4} e^{j \omega_{e} T_{0}}+c c, \\
& x_{50}=\eta_{1} A_{1}\left(T_{1}\right) e^{i \omega_{1} T_{0}}+\eta_{2} A_{2}\left(T_{1}\right) e^{i \omega_{2} T_{0}} \\
& +\eta_{3} A_{3}\left(T_{1}\right) e^{i \omega_{3} T_{0}}+\eta_{4} A_{4}\left(T_{1}\right) e^{i \omega_{4} T_{0}} \\
& +\eta_{5} A_{5}\left(T_{1}\right) e^{i \omega_{5} T_{0}}+\rho_{5} e^{j \omega_{e} T_{0}}+c c,
\end{aligned}
$$

where $\rho_{1}=A_{\mathrm{N} 11} B_{1}+A_{\mathrm{N} 12} B_{2}+A_{\mathrm{N} 13} B_{3}+A_{\mathrm{N} 14} B_{4}+A_{\mathrm{N} 15} B_{5}$, $\rho_{2}=A_{\mathrm{N} 21} B_{1}+A_{\mathrm{N} 22} B_{2}+A_{\mathrm{N} 23} B_{3}+A_{\mathrm{N} 24} B_{4}+A_{\mathrm{N} 25} B_{5}, \rho_{3}=$ $A_{\mathrm{N} 31} B_{1}+A_{\mathrm{N} 32} B_{2}+A_{\mathrm{N} 33} B_{3}+A_{\mathrm{N} 34} B_{4}+A_{\mathrm{N} 35} B_{5}, \rho_{4}=A_{\mathrm{N} 41} B_{1}+$ $A_{\mathrm{N} 42} B_{2}+A_{\mathrm{N} 43} B_{3}+A_{\mathrm{N} 44} B_{4}+A_{\mathrm{N} 45} B_{5}$, and $\rho_{5}=A_{\mathrm{N} 51} B_{1}+$ $A_{\mathrm{N} 52} B_{2}+A_{\mathrm{N} 53} B_{3}+A_{\mathrm{N} 54} B_{4}+A_{\mathrm{N} 55} B_{5}$.

After substituting (35) into (32), there are multiple frequency components such as $2 \omega_{j}, \pm\left(\omega_{j} \pm \omega_{k}\right), 2 \omega_{e}$, and $\pm\left(\omega_{j} \pm\right.$ $\left.\omega_{e}\right)$, where $k=1,2,3,4,5$ and $k \neq j$. When there is no internal resonance, the secular terms can be eliminated from (32) to obtain

$$
\begin{aligned}
-c_{\mathrm{N} m} A_{m}-2 \dot{A}_{m} & =0, \\
-c_{\mathrm{N} 4} i \omega_{4} A_{4} e^{i \omega_{4} t}-2 i \omega_{4} \dot{A}_{4} e^{i \omega_{4} t}+L_{4} e^{2 i \omega_{e} T_{0}} & =0 .
\end{aligned}
$$

The solutions of (36) are written as

$$
\begin{aligned}
& A_{m}=E_{m} e^{-c_{\mathrm{N} m}^{\prime} t / 2}, \\
& A_{4}=E_{4} e^{-c_{\mathrm{N} 4}^{\prime} t / 2}-\frac{L_{4} e^{i\left(\varepsilon \sigma_{2} t+\varphi_{2}\right)}}{\omega_{4} \sqrt{c_{\mathrm{N} 4}^{2}+4 \varepsilon^{2} \sigma_{2}^{2}}},
\end{aligned}
$$

where $\varphi_{2}$ is a constant related to $\varepsilon$ and $\sigma, \cos \varphi_{2}=2 \varepsilon \sigma_{2} /$ $\sqrt{c_{\mathrm{N} 4}^{\prime 2}+4 \varepsilon^{2} \sigma_{2}^{2}}$, and $\sin \varphi_{2}=c_{\mathrm{N} 4} / \sqrt{c_{\mathrm{N} 4}^{\prime 2}+4 \varepsilon^{2} \sigma_{2}^{2}}$.

Because of the damping in the EIMG system, the initial vibration displacements will decrease to zero. Under the normal coordinate system, the zero-order stable analytical solutions are given by

$$
\begin{aligned}
& x_{\mathrm{N} m 0}=B_{m} e^{i \omega_{e} T_{0}}, \\
& x_{\mathrm{N} 40}=-\frac{L_{4} e^{i\left(\omega_{4} t+\varepsilon \sigma_{2} t+\theta_{2}\right)}}{\omega_{4} \sqrt{c_{\mathrm{N} 4}^{\prime 2}+4 \varepsilon^{2} \sigma_{2}^{2}}}+B_{4} e^{i \omega_{e} T_{0}}+c c .
\end{aligned}
$$

After eliminating the secular terms, the first-order approximate solutions are obtained by substituting (38) into (32):

$$
\begin{aligned}
& x_{\mathrm{N} m 1}=\frac{V_{4} e^{2 i \omega_{e} t}}{\omega_{m}^{2}-4 \omega_{e}^{2}} \\
& +\frac{T_{m} L_{4}^{2} e^{2 i\left(\omega_{4} t+\varepsilon \sigma_{2} t+\varphi_{2}\right)}}{\omega_{4}^{2}\left(c_{\mathrm{N} 4}^{\prime 2}+4 \varepsilon^{2} \sigma_{2}^{2}\right)\left[\omega_{m}^{2}-4\left(\omega_{4}+\varepsilon \sigma_{2}\right)^{2}\right]} \\
& -\frac{U_{m} L_{4}}{\omega_{4} \sqrt{c_{\mathrm{N} 4}^{\prime 2}+4 \varepsilon^{2} \sigma_{2}^{2}}}\left[\frac{e^{i\left[\left(\omega_{4}+\omega_{e}+\varepsilon \sigma_{2}\right) t+\varphi_{2}\right]}}{\omega_{m}^{2}-\left(\omega_{4}+\omega_{e}+\varepsilon \sigma_{2}\right)^{2}}\right. \\
& \left.+\frac{e^{i\left[\left(\omega_{e}-\omega_{4}+\varepsilon \sigma_{2}\right) t+\varphi_{2}\right]}}{\omega_{m}^{2}-\left(\omega_{e}-\omega_{4}+\varepsilon \sigma_{2}\right)^{2}}\right], \\
& x_{\mathrm{N} 41}=\frac{T_{4} L_{4}^{2} e^{2 i\left(\omega_{4} t+\varepsilon \sigma_{2} t+\varphi_{2}\right)}}{\omega_{4}^{2}\left(c_{\mathrm{N} 4}^{\prime 2}+4 \varepsilon^{2} \sigma_{2}^{2}\right)\left[\omega_{4}^{2}-4\left(\omega_{4}+\varepsilon \sigma_{2}\right)^{2}\right]} \\
& +\frac{V_{4} e^{2 i \omega_{e} t}}{\omega_{4}^{2}-4 \omega_{e}^{2}} \\
& -\frac{U_{4} L_{4}}{\omega_{4} \sqrt{c_{\mathrm{N} 4}^{\prime 2}+4 \varepsilon^{2} \sigma_{2}^{2}}}\left[\frac{e^{i\left[\left(\omega_{4}+\omega_{e}+\varepsilon \sigma_{2}\right) t+\varphi_{2}\right]}}{\omega_{4}^{2}-4\left(\omega_{4}+\omega_{e}+\varepsilon \sigma_{2}\right)^{2}}\right. \\
& \left.+\frac{e^{i\left[\left(\omega_{e}-\omega_{4}+\varepsilon \sigma_{2}\right) t+\varphi_{2}\right]}}{\omega_{4}^{2}-4\left(\omega_{e}-\omega_{4}+\varepsilon \sigma_{2}\right)^{2}}\right],
\end{aligned}
$$

where $T_{i}=A_{\mathrm{N} 1 i} P_{1}+A_{\mathrm{N} 2 i}\left(P_{1}+P_{2}\right)+A_{\mathrm{N} 3 i}\left(P_{2}+P_{3}\right)+A_{\mathrm{N} 4 i}\left(P_{3}+\right.$ $\left.P_{4}\right)+A_{\mathrm{N} 5 i} P_{5}, U_{i}=A_{\mathrm{N} 1 i} Q_{1}+A_{\mathrm{N} 2 i}\left(Q_{1}+Q_{2}\right)+A_{\mathrm{N} 3 i}\left(Q_{2}+Q_{3}\right)+$ $A_{\mathrm{N} 4 i}\left(Q_{3}+Q_{4}\right)+A_{\mathrm{N} 5 i} Q_{5}, V_{i}=A_{\mathrm{N} 1 i} S_{1}+A_{\mathrm{N} 2 i}\left(S_{1}+S_{2}\right)+A_{\mathrm{N} 3 i}\left(S_{2}+\right.$ $\left.S_{3}\right)+A_{\mathrm{N} 4 i}\left(S_{3}+S_{4}\right)+A_{\mathrm{N} 5 i} S_{5}, P_{1}=-\left(c_{1} \mu_{1}^{2}+d_{1} \mu_{2}^{2}+e_{1} \mu_{1} \mu_{2}\right), P_{2}=$ $-\left(c_{2} \mu_{3}^{2}+d_{2} \mu_{2}^{2}+e_{2} \mu_{2} \mu_{3}\right), P_{3}=-\left(c_{3} \mu_{3}^{2}+d_{3} \mu_{4}^{2}+e_{3} \mu_{3} \mu_{4}\right)$, and $P_{4}=-\left(c_{4} \mu_{5}^{2}+d_{4} \mu_{4}^{2}+e_{4} \mu_{4} \mu_{5}\right) ; Q_{1}=-\left[2 c_{1} \mu_{1} \rho_{1}+2 d_{1} \mu_{2} \rho_{2}+\right.$ $\left.e_{1}\left(\rho_{1} \mu_{2}+\rho_{2} \mu_{1}\right)\right], Q_{2}=-\left[2 c_{2} \mu_{3} \rho_{3}+2 d_{2} \mu_{2} \rho_{2}+e_{2}\left(\rho_{2} \mu_{3}+\right.\right.$ $\left.\left.\rho_{3} \mu_{2}\right)\right], Q_{3}=-\left[2 c_{3} \mu_{3} \rho_{3}+2 d_{3} \mu_{4} \rho_{4}+e_{3}\left(\rho_{3} \mu_{4}+\rho_{4} \mu_{3}\right)\right]$, and $Q_{4}=-\left[2 c_{4} \mu_{5} \rho_{5}+2 d_{4} \mu_{4} \rho_{4}+e_{4}\left(\rho_{4} \mu_{5}+\rho_{5} \mu_{4}\right)\right]$; and $S_{1}=$ $-\left(c_{1} \rho_{1}^{2}+d_{1} \rho_{2}^{2}+e_{1} \rho_{1} \rho_{2}\right), S_{2}=-\left(c_{2} \rho_{3}^{2}+d_{2} \rho_{2}^{2}+e_{2} \rho_{2} \rho_{3}\right), S_{3}=$ $-\left(c_{3} \rho_{3}^{2}+d_{3} \rho_{4}^{2}+e_{3} \rho_{3} \rho_{4}\right)$, and $S_{4}=-\left(c_{4} \rho_{5}^{2}+d_{4} \rho_{4}^{2}+e_{4} \rho_{4} \rho_{5}\right)$.

Under the normal coordinate system, the approximate solutions are then calculated by substituting (38) and (39) into (29):

$$
\begin{aligned}
x_{\mathrm{N} m} & =B_{m} e^{i \omega_{e} T_{0}}+\frac{\varepsilon V_{m} e^{2 i \omega_{e} t}}{\omega_{m}^{2}-4 \omega_{e}^{2}} \\
+ & \frac{\varepsilon T_{m} Y_{4}^{2} e^{2 i\left(\omega_{4} t+\varepsilon \sigma_{2} t+\varphi_{2}\right)}}{\omega_{4}^{2}\left(c_{\mathrm{N} 4}^{\prime 2}+4 \varepsilon^{2} \sigma_{2}^{2}\right)\left[\omega_{m}^{2}-4\left(\omega_{4}+\varepsilon \sigma_{2}\right)^{2}\right]}
\end{aligned}
$$




$$
\begin{gathered}
-\frac{\varepsilon U_{m} L_{4}}{\omega_{4} \sqrt{c_{\mathrm{N} 4}^{\prime 2}+4 \varepsilon^{2} \sigma_{2}^{2}}}\left[\frac{e^{i\left[\left(\omega_{4}+\omega_{e}+\varepsilon \sigma_{2}\right) t+\varphi_{2}\right]}}{\omega_{m}^{2}-\left(\omega_{4}+\omega_{e}+\varepsilon \sigma_{2}\right)^{2}}\right. \\
\left.+\frac{e^{i\left[\left(\omega_{e}-\omega_{4}+\varepsilon \sigma_{2}\right) t+\varphi_{2}\right]}}{\omega_{m}^{2}-\left(\omega_{e}-\omega_{4}+\varepsilon \sigma_{2}\right)^{2}}\right]+c c, \\
x_{\mathrm{N} 4}=B_{4} e^{i \omega_{e} T_{0}}-\frac{L_{4} e^{i\left(\omega_{4} t+\varepsilon \sigma_{2} t+\varphi_{2}\right)}}{\omega_{4} \sqrt{\mathcal{c}_{\mathrm{N} 4}^{\prime 2}+4 \varepsilon^{2} \sigma_{2}^{2}}} \\
+\frac{\varepsilon T_{4} L_{4}^{2} e^{2 i\left(\omega_{4} t+\varepsilon \sigma_{2} t+\varphi_{2}\right)}}{\omega_{4}^{2}\left(c_{\mathrm{N} 4}^{\prime 2}+4 \varepsilon^{2} \sigma_{2}^{2}\right)\left[\omega_{4}^{2}-4\left(\omega_{4}+\varepsilon \sigma_{2}\right)^{2}\right]} \\
+\frac{\varepsilon V_{4} e^{2 i \omega_{e} t}}{\omega_{4}^{2}-4 \omega_{e}^{2}} \\
+\frac{\varepsilon U_{4} L_{4}}{\omega_{4} \sqrt{\mathcal{c}_{\mathrm{N} 4}^{\prime 2}+4 \varepsilon^{2} \sigma_{2}^{2}}}\left[\frac{e^{i\left[\left(\omega_{4}+\omega_{e}+\varepsilon \sigma_{2}\right) t+\varphi_{2}\right]}}{\omega_{4}^{2}-\left(\omega_{4}+\omega_{e}+\varepsilon \sigma_{2}\right)^{2}}\right. \\
\left.+\frac{e^{i\left[\left(\omega_{e}-\omega_{4}+\varepsilon \sigma_{2}\right) t+\varphi_{2}\right]}}{\omega_{4}^{2}-\left(\omega_{e}-\omega_{4}+\varepsilon \sigma_{2}\right)^{2}}\right]+c c . \\
]
\end{gathered}
$$

When the wave frequency of the torque wave is close to half of the natural frequency of the EIMG system, namely, $2 \omega_{e}=\omega_{j}+\varepsilon \sigma_{2}$, the approximate solutions of the EIMG system under the normal coordinate system are

$$
\begin{aligned}
x_{\mathrm{N} m} & =B_{m} e^{i \omega_{e} T_{0}}+\frac{\varepsilon V_{m} e^{2 i \omega_{e} t}}{\omega_{m}^{2}-4 \omega_{e}^{2}} \\
+ & \frac{\varepsilon T_{i} L_{i}^{2} e^{2 i\left(\omega_{k} t+\varepsilon \sigma_{2} t+\varphi_{2}\right)}}{\omega_{k}^{2}\left(c_{\mathrm{N} k}^{\prime 2}+4 \varepsilon^{2} \sigma_{2}^{2}\right)\left[\omega_{m}^{2}-4\left(\omega_{k}+\varepsilon \sigma_{2}\right)^{2}\right]} \\
- & \frac{\varepsilon U_{m} L_{i}}{\omega_{k} \sqrt{c_{\mathrm{N} k}^{\prime 2}+4 \varepsilon^{2} \sigma_{2}^{2}}}\left[\frac{e^{i\left[\left(\omega_{k}+\omega_{e}+\varepsilon \sigma_{2}\right) t+\varphi_{2}\right]}}{\omega_{m}^{2}-\left(\omega_{k}+\omega_{e}+\varepsilon \sigma_{2}\right)^{2}}\right. \\
+ & \left.\frac{e^{i\left[\left(\omega_{e}-\omega_{k}+\varepsilon \sigma_{2}\right) t+\varphi_{2}\right]}}{\omega_{m}^{2}-\left(\omega_{e}-\omega_{k}+\varepsilon \sigma_{2}\right)^{2}}\right]+c c, \\
x_{\mathrm{N} i} & =B_{k} e^{i \omega_{e} T_{0}}-\frac{L_{k} e^{i\left(\omega_{k} t+\varepsilon \sigma_{2} t+\varphi_{2}\right)}}{\omega_{k} \sqrt{c_{\mathrm{N} k}^{2}+4 \varepsilon^{2} \sigma_{2}^{2}}} \\
+ & \left.\frac{\varepsilon e^{i\left[\left(\omega_{e}-\omega_{k}+\varepsilon \sigma_{2}\right) t+\varphi_{2}\right]}}{\omega_{k}^{2}-\left(\omega_{e}-\omega_{k}+\varepsilon \sigma_{2}\right)^{2}}\right]+c c . e^{2 i\left(\omega_{k} t+\varepsilon \sigma_{2} t+\varphi_{2}\right)} \\
+ & \frac{\varepsilon V_{k} e^{2 i \omega_{e} t}}{\omega_{k}^{2}-4 \omega_{e}^{2}} \\
& \frac{\left.\varepsilon U_{k}^{\prime 2} L_{k}+4 \varepsilon^{2} \sigma_{2}^{2}\right)\left[\omega_{k}^{2}-4\left(\omega_{k}+\varepsilon \sigma_{2}\right)^{2}\right]}{\omega_{\mathrm{N} k}^{\prime 2}+4 \varepsilon^{2} \sigma_{2}^{2}}\left[\frac{e^{i\left[\left(\omega_{k}+\omega_{e}+\varepsilon \sigma_{2}\right) t+\varphi_{2}\right]}}{\omega_{k}^{2}-\left(\omega_{k}+\omega_{e}+\varepsilon \sigma_{2}\right)^{2}}\right.
\end{aligned}
$$

The superharmonic resonance responses of the EIMG system under the regular coordinate system are calculated using (28).

The initial displacements $E_{k}$ are obtained from the static solution of the linear differential equations derived for the EIMG system. The linear differential equations in (7) are expressed as

$$
\mathbf{M} \ddot{\mathbf{x}}+\mathbf{k x}=\mathbf{F}_{0},
$$

where $\mathbf{F}_{0}=\left[\begin{array}{lllll}T_{1} / R_{\mathrm{s}} & T_{2} / R_{\mathrm{If}} & T_{3} / R_{\mathrm{I}} & T_{4} / R_{\text {of }} & T_{5} / R_{\mathrm{o}}\end{array}\right]^{T}$ and $T_{2}$, $T_{4}$ are the output torques on the inner and outer FP, respectively.

By setting the mass matrix in (42) equal to zero and only considering the static loads, the static displacement equations can be expressed under the assumption that the deformations of components caused by gravity and the stiffness waves are negligible. In this case, we have

$$
\mathbf{k x}_{0}=\mathbf{F}_{0}
$$

where $\mathbf{x}_{0}=\left[\begin{array}{lllll}E_{1} & E_{2} & E_{3} & E_{4} & E_{5}\end{array}\right]^{T}$.

\section{Resonance of Example EIMG System and Discussion}

A finite element model (FEM) of the example EIMG system described in Table 1 can be built using Ansys; a diagram of the system is presented in Figure 3. In this model, the inner stator and the permeable magnetic of the inner and outer FP consist of silicon steel (23TW250) sheets. The back irons of the inner rotor and outer stator are also made of 23 TW250. The nonpermeable magnets of the inner and outer FP are made of epikote, which has similar material characteristics to air. The remanence and coercivity of the PMs on the inner rotor and outer stator are coincident (see Table 1). In this study, we consider linear and unsaturated magnetic circuits. The air gaps have denser grids because these areas have greater magnetic reluctance and higher variation gradients of magnetic flux density than the PMs and back irons. The torque profiles calculated for all components are displayed in Figure 4.

Figure 4 indicates that the torques on all components vary sinusoidally with respect to the phase angle. The wave period is determined by the number of pole pairs of the currents on the inner stator or the number of pole pairs of the PMs on the inner rotor and outer stator; that is, $T_{i}=2 \pi / p_{i}$. The torque profiles can be expressed as $T_{c i}=\sin \left(p_{i} \theta_{i j}+\theta_{i 0}\right)$, where $\theta_{i j}$ is the relative torsional displacement between two adjacent components. When the outer torque on the outer FP is less than the maximum $T_{c 3}$ and takes a constant value, $\theta_{i 0}$ is also constant. As such, $T_{c i}$ is a (nonlinear) sinusoidal function of the relative torsional displacements.

Table 2 shows the corresponding dynamic parameters of the example EIMG system.

By substituting the dynamic parameters of the example system into (25) and (28), the main resonance responses of the EIMG system can be determined. When the wave frequency is close to the natural frequency of the outer FP 

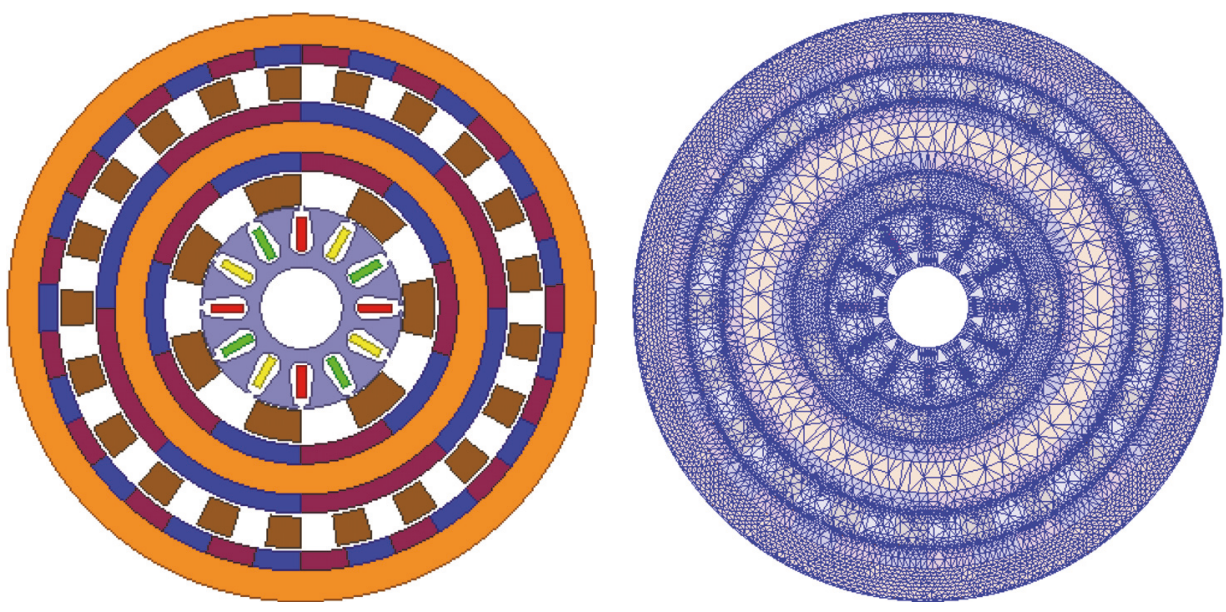

FIGURE 3: FEM of the example EIMG system.

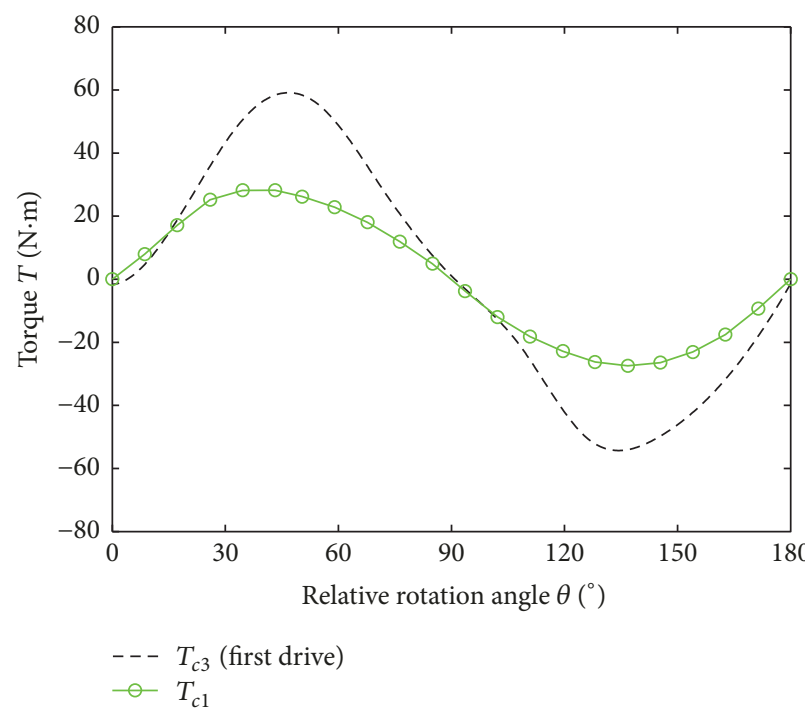

(a)

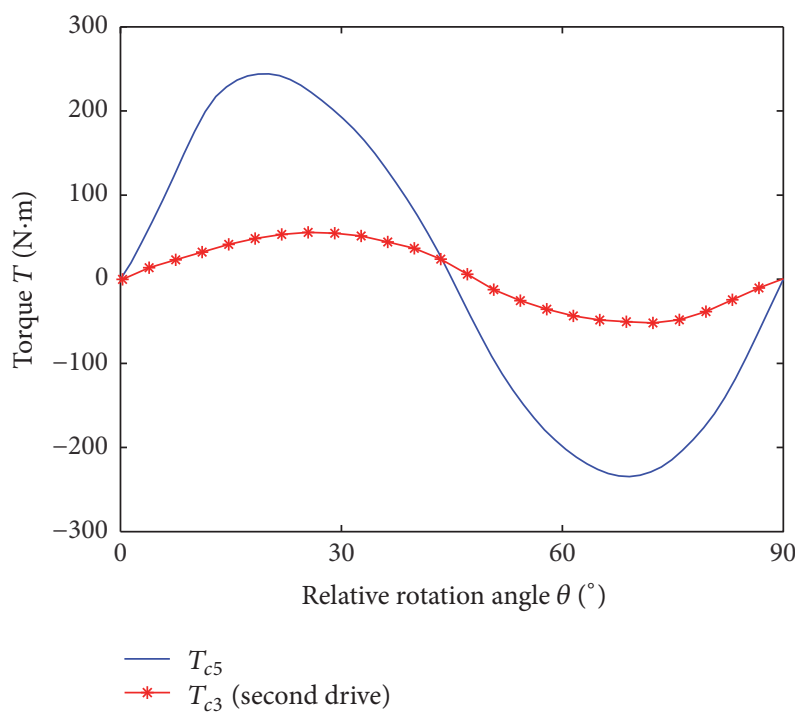

(b)

FIGURE 4: Torque profiles of the example EIMG system.

TABle 1: Parameters of example EIMG system.

\begin{tabular}{|c|c|c|c|}
\hline Number of pole pairs on inner stator & 2 & Number of pole pairs on inner surface of inner rotor & 5 \\
\hline Number of pole pairs on outer surface of inner rotor & 4 & Number of pole pairs on outer stator & 17 \\
\hline Number of inner FP & 7 & Number of outer FP & 21 \\
\hline Outer radius of inner stator $/ \mathrm{mm}$ & 98 & Inner radius of inner $\mathrm{FP} / \mathrm{mm}$ & 100 \\
\hline Outer radius of inner $\mathrm{FP} / \mathrm{mm}$ & 130 & Thickness of PMs on inner rotor/mm & 10 \\
\hline Inner radius of inner rotor yoke $/ \mathrm{mm}$ & 152 & Outer radius of inner rotor yoke/mm & 182 \\
\hline Inner radius of outer $\mathrm{FP} / \mathrm{mm}$ & 204 & Outer radius of outer $\mathrm{FP} / \mathrm{mm}$ & 234 \\
\hline Thickness of PMs on outer stator/mm & 10 & Inner radius of outer stator yoke $/ \mathrm{mm}$ & 256 \\
\hline Outer radius of outer stator yoke/mm & 276 & Axial length/mm & 40 \\
\hline Remanence of PMs/T & 1.3 & Coercive force of $\mathrm{PMs} / \mathrm{KOe}$ & 11.6 \\
\hline Magnitude of currents on inner stator/A & 20 & & \\
\hline
\end{tabular}


TABLE 2: Characteristic parameters of example EIMG system.

\begin{tabular}{lcccccc}
\hline$k_{\mathrm{s}}(\mathrm{N} / \mathrm{m})$ & $k_{\mathrm{If}}(\mathrm{N} / \mathrm{m})$ & $K_{\mathrm{o}}(\mathrm{N} / \mathrm{m})$ & $\theta_{10}(\mathrm{rad})$ & $\theta_{20}(\mathrm{rad})$ & $M_{1}(\mathrm{~kg})$ & $M_{2}(\mathrm{~kg})$ \\
\hline $8.6444 \times 10^{5}$ & $3.1446 \times 10^{5}$ & $4.5467 \times 10^{5}$ & $5 \pi / 12$ & $\pi / 4$ & 0.64 & 0.657 \\
\hline$M_{3}(\mathrm{~kg})$ & $M_{4}(\mathrm{~kg})$ & $M_{5}(\mathrm{~kg})$ & $\zeta_{\mathrm{s}}$ & $\zeta_{\text {If }}$ & $\zeta_{\mathrm{I}}$ & $\zeta_{\text {of }}$ \\
\hline 5.4 & 3.0 & 3.85 & 0.057 & 0.0653 & 0.019 & 0.0173 \\
\hline$\zeta_{0}$ & $T_{1}(\mathrm{~N} \cdot \mathrm{m})$ & $T_{2}(\mathrm{~N} \cdot \mathrm{m})$ & $T_{3}(\mathrm{~N} \cdot \mathrm{m})$ & $T_{4}(\mathrm{~N} \cdot \mathrm{m})$ & $T_{5}(\mathrm{~N} \cdot \mathrm{m})$ & $\Delta T(\mathrm{~N} \cdot \mathrm{m})$ \\
\hline 0.0793 & 22 & 77 & 55 & 288.75 & 233.75 & 1 \\
\hline
\end{tabular}

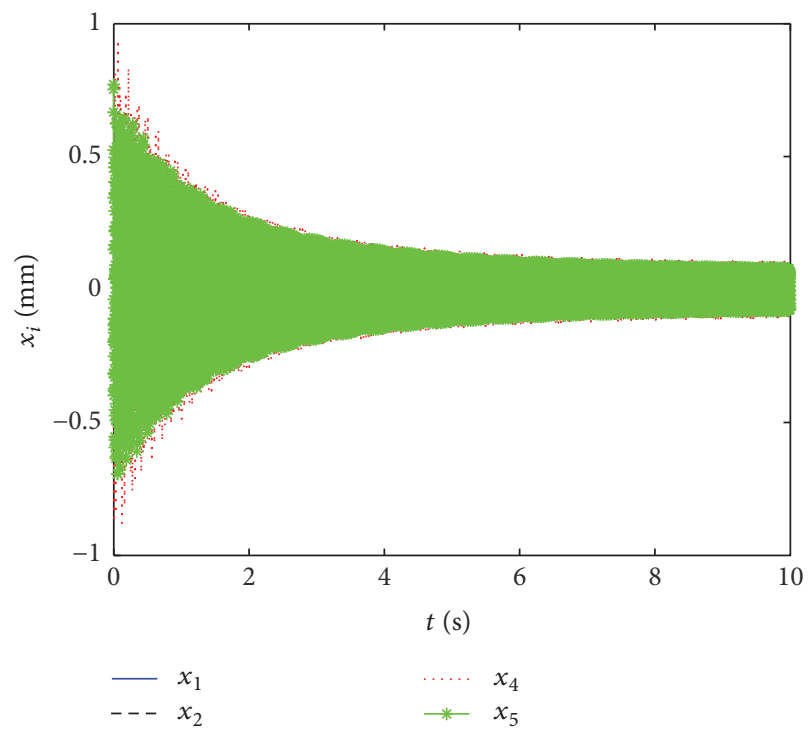

(a)

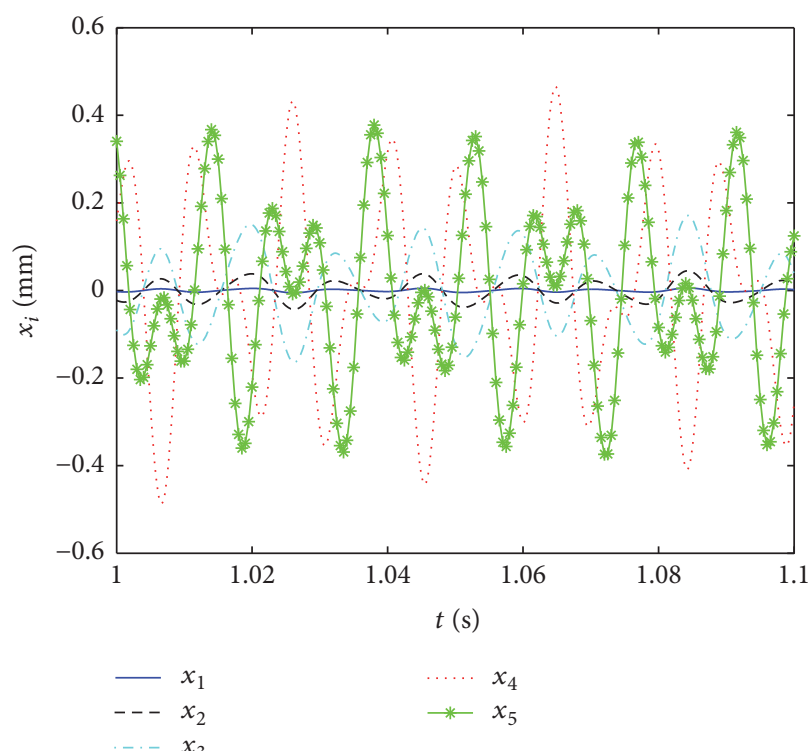

(b)

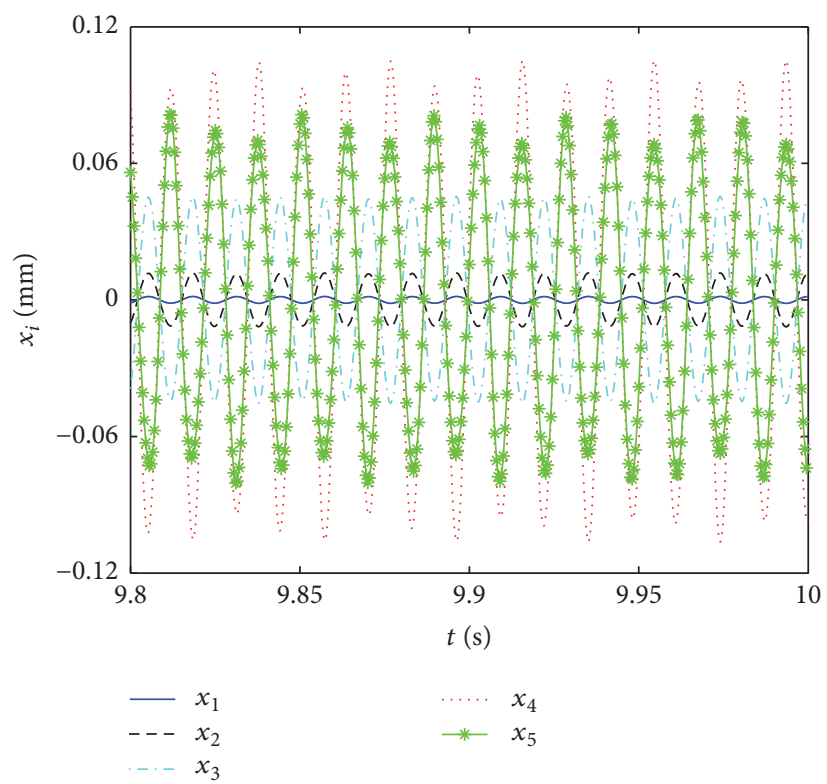

(c)

FIGURE 5: Transient responses of EIMG system when $\omega_{e} \approx \omega_{4}$.

rotational mode, the transient responses of the components are shown in Figure 5.

Figure 5 indicates that the output torque causes larger initial static displacements among the components of the EIMG system. The system damping ensures that the transient responses will gradually transition to the steady state. This process takes approximately $6 \mathrm{~s}$. When resonance occurs, there are multiple frequency components in the transient responses, and the steady-state responses have a particular frequency. 

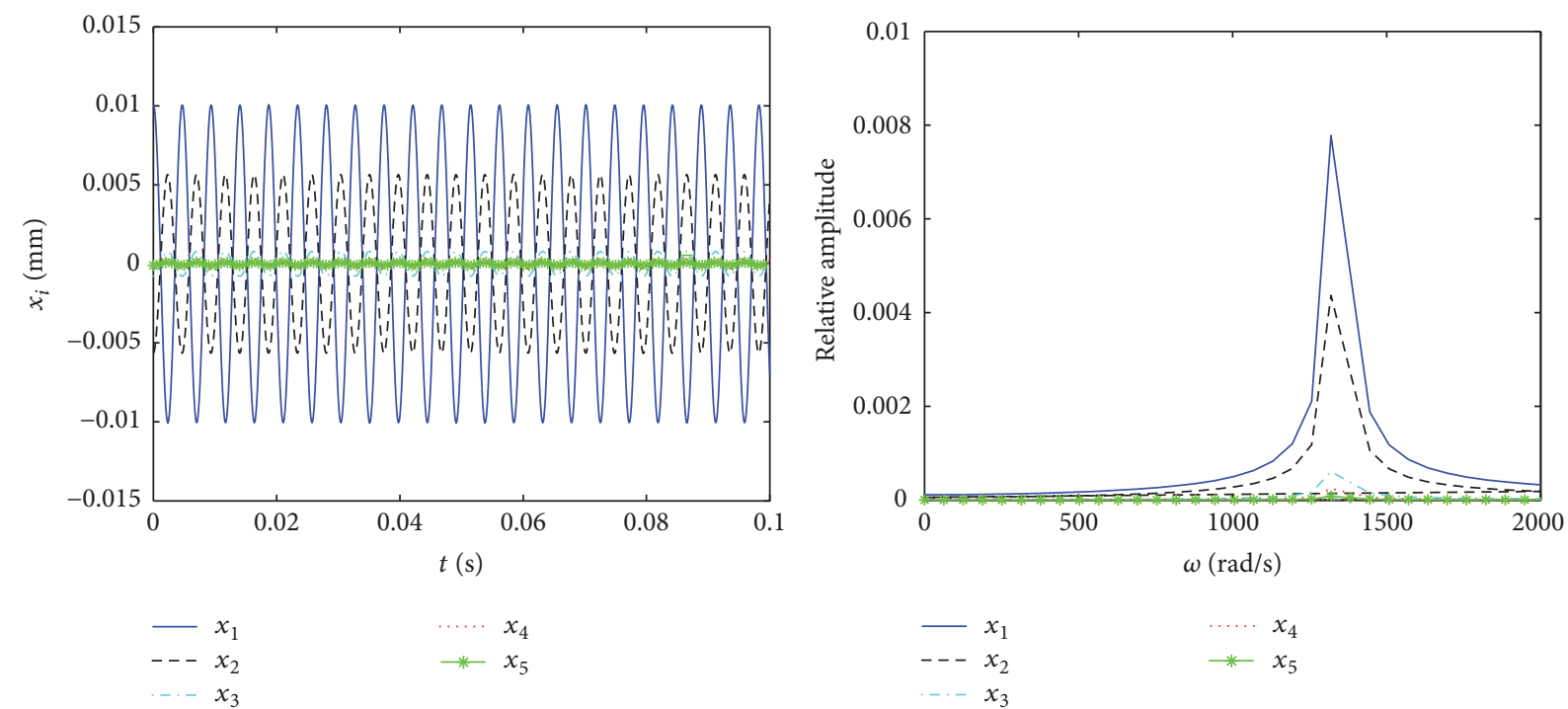

(a) $\omega_{e} \approx \omega_{1}$
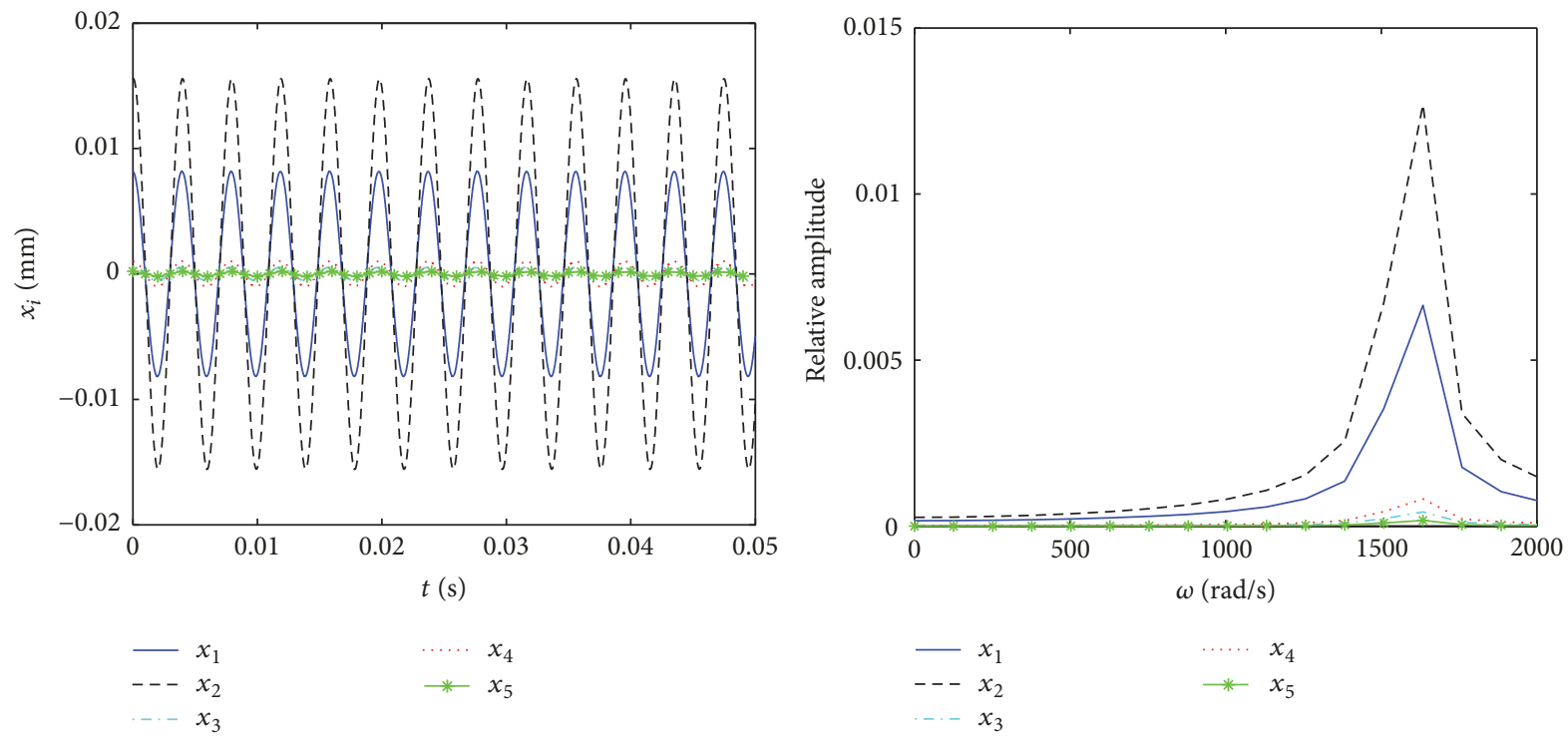

(b) $\omega_{e} \approx \omega_{2}$
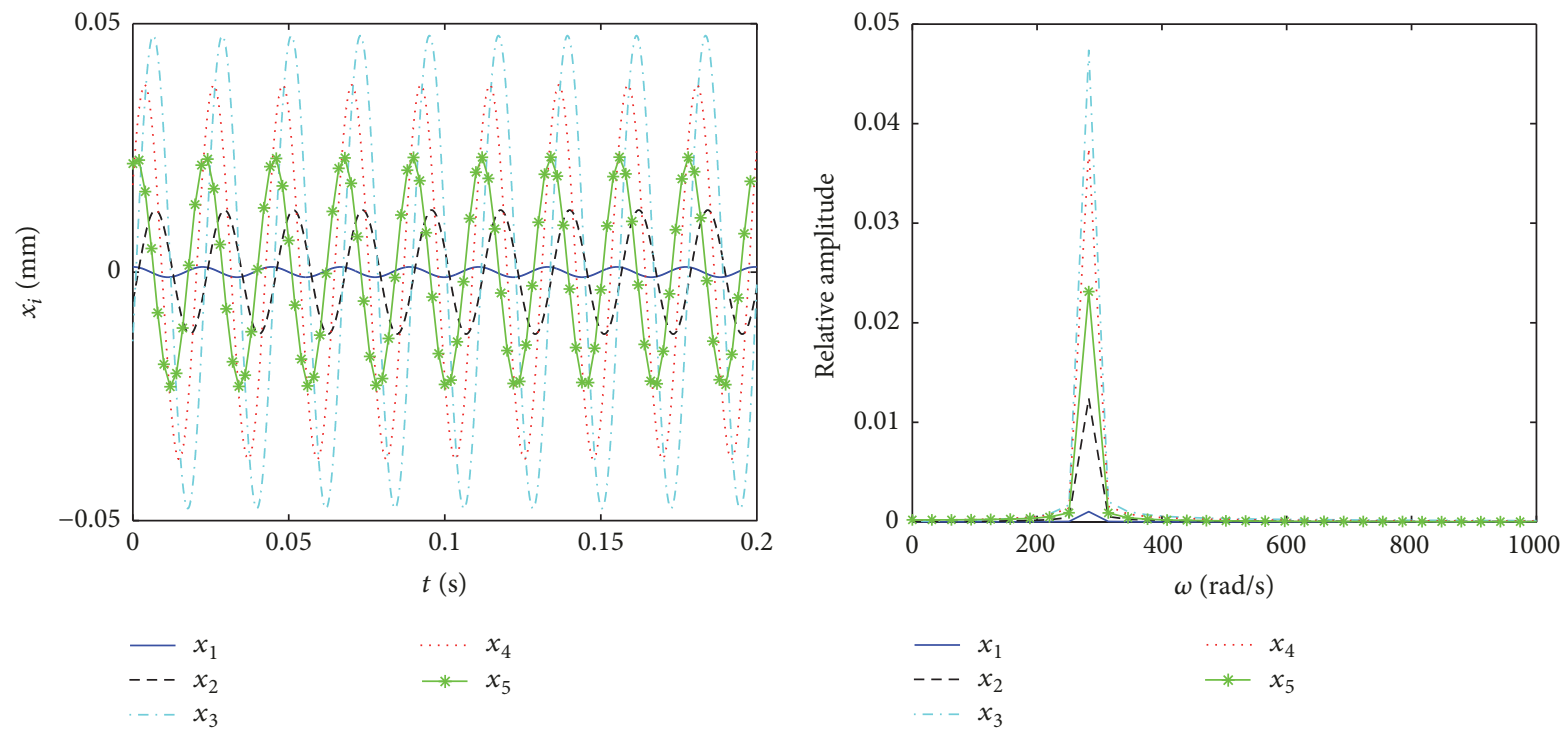

(c) $\omega_{e} \approx \omega_{3}$

Figure 6: Continued. 

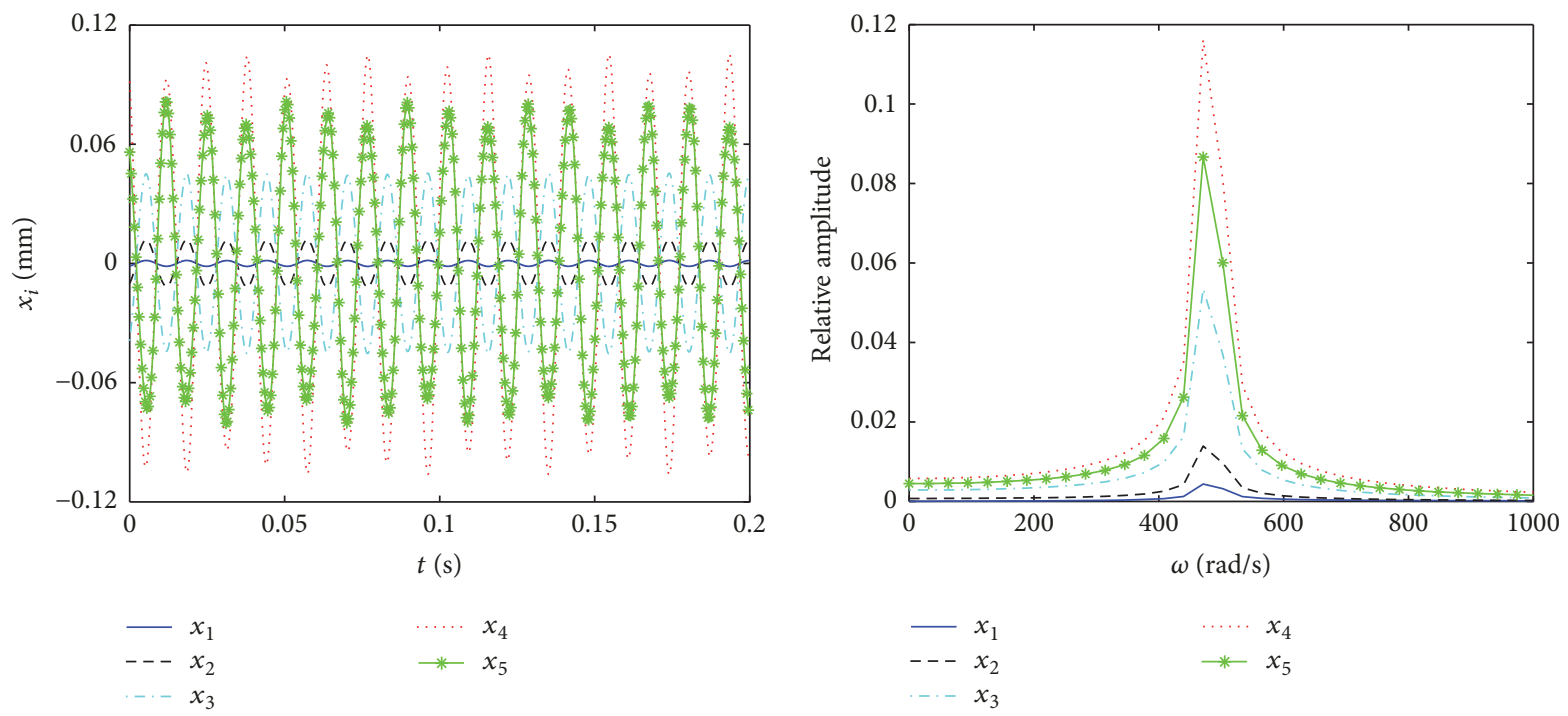

(d) $\omega_{e} \approx \omega_{4}$
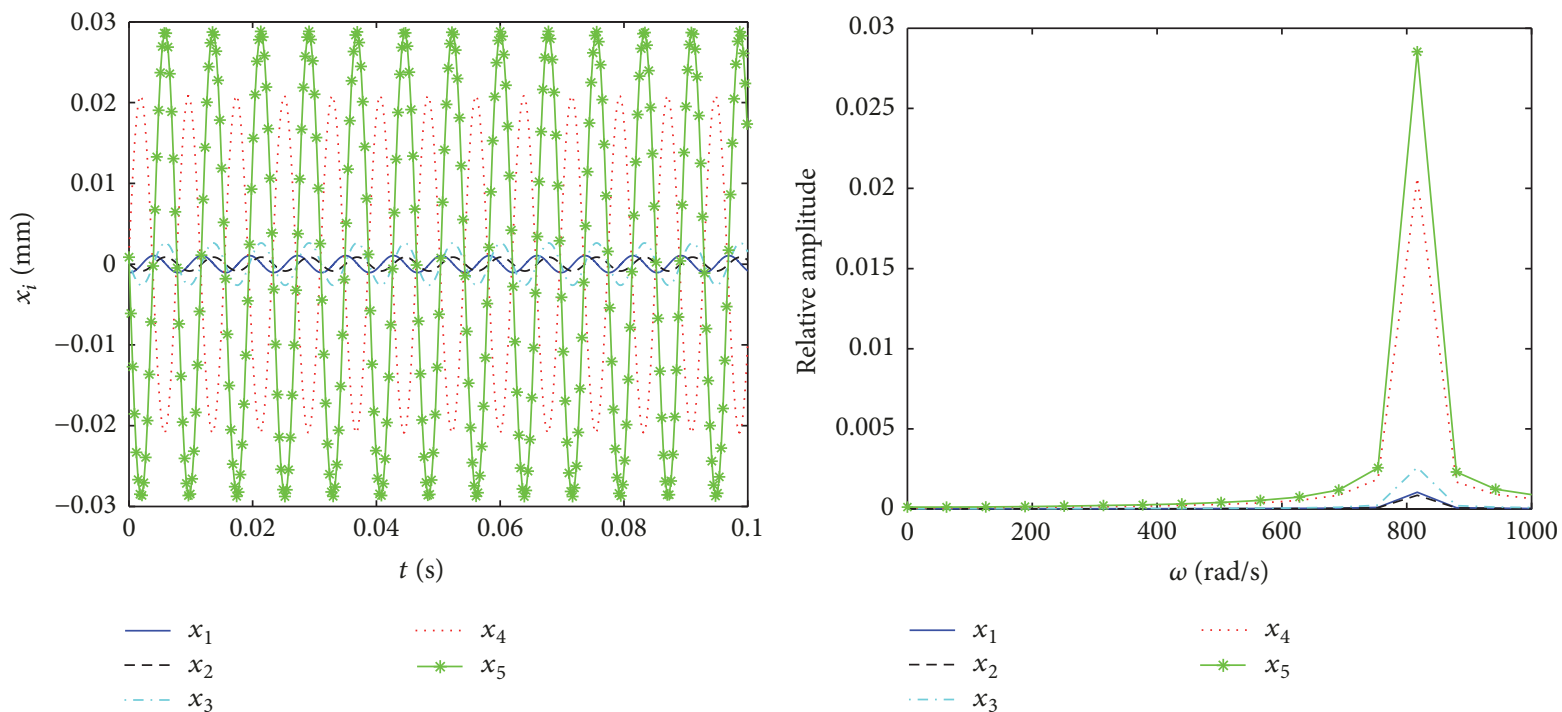

(e) $\omega_{e} \approx \omega_{5}$

FIgURE 6: Main resonance when $\omega_{e} \approx \omega_{i}$.

Because of the magnetic coupling among components in the EIMG system, the magnetic coupling stiffness is much smaller than the mechanical support stiffness. Moreover, because there is no contact among the components (namely, no frictional damping), the resonance amplitudes of the transient responses will be larger and will slowly decay to zero. This results in some deterioration in the dynamic characteristics.

Considering the effects of damping, the resonance will gradually be close to stabilization. When the wave frequency $\omega_{e}$ is close to the natural frequency $\omega_{i}$ and half of the corresponding frequency $\omega_{i} / 2$ of the derived nonlinear EIMG system, the steady-state response curves are as shown in Figures 6 and 7.

Figures 6 and 7 show that strong resonance will occur when the wave frequency is close to the natural frequency or half of the natural frequency of the derived EIMG system. The dominant frequency in the superharmonic resonance is the natural frequency, rather than the wave frequency or half of the natural frequency. The resonance amplitudes are much larger when the wave frequency is close to the natural frequency of the inner rotor rotational mode and outer FP rotational mode than when the wave frequency is close to the natural frequency of the inner FP rotational mode and inner stator rotational mode.

When resonance occurs and the displacement of a certain degree of freedom reaches its maximum, the resonance amplitudes are very different. For instance, when the wave frequency is close to the natural frequency of the outer FP rotational mode, the torsional displacements of the outer FP attain their maximum. Furthermore, the displacements of the adjacent components, namely, the inner rotor and outer 

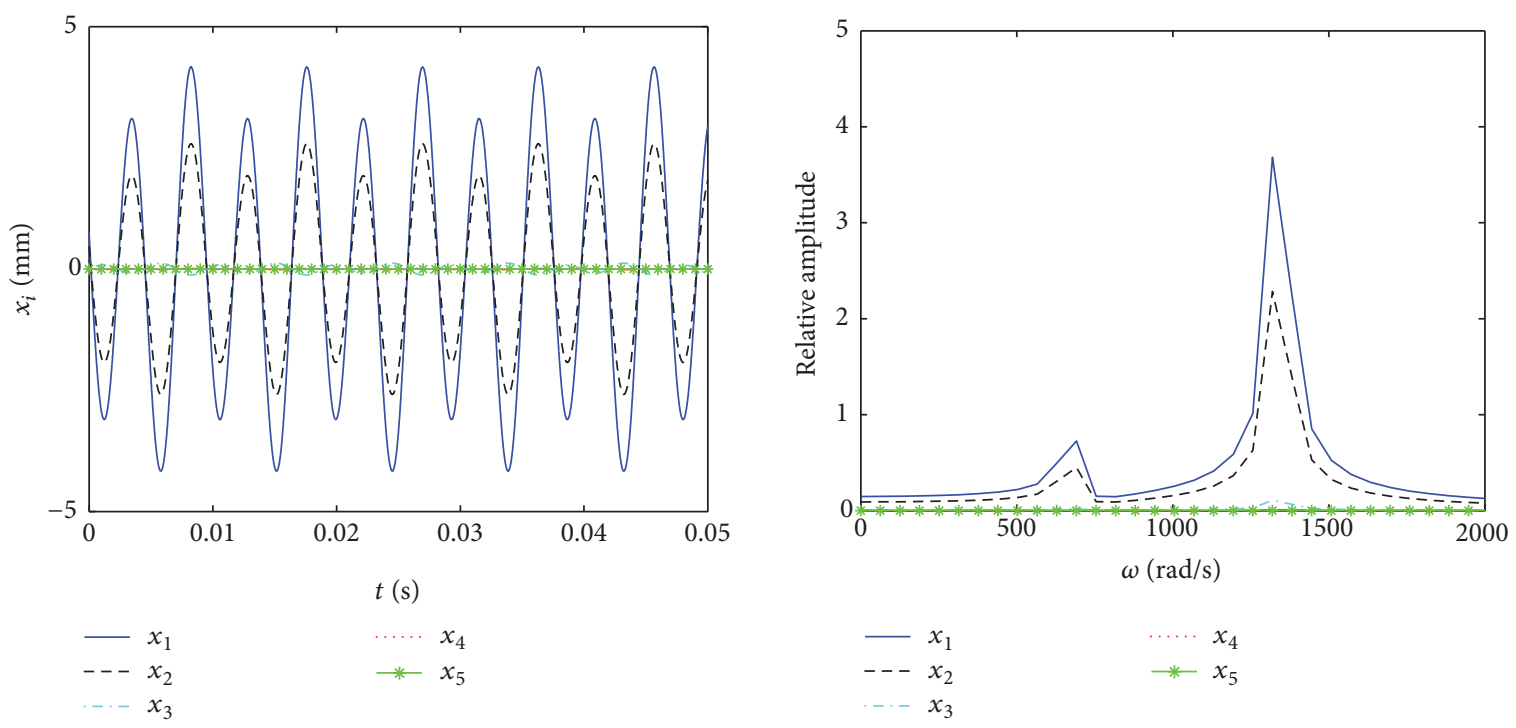

(a) $\omega_{e} \approx \omega_{1} / 2$
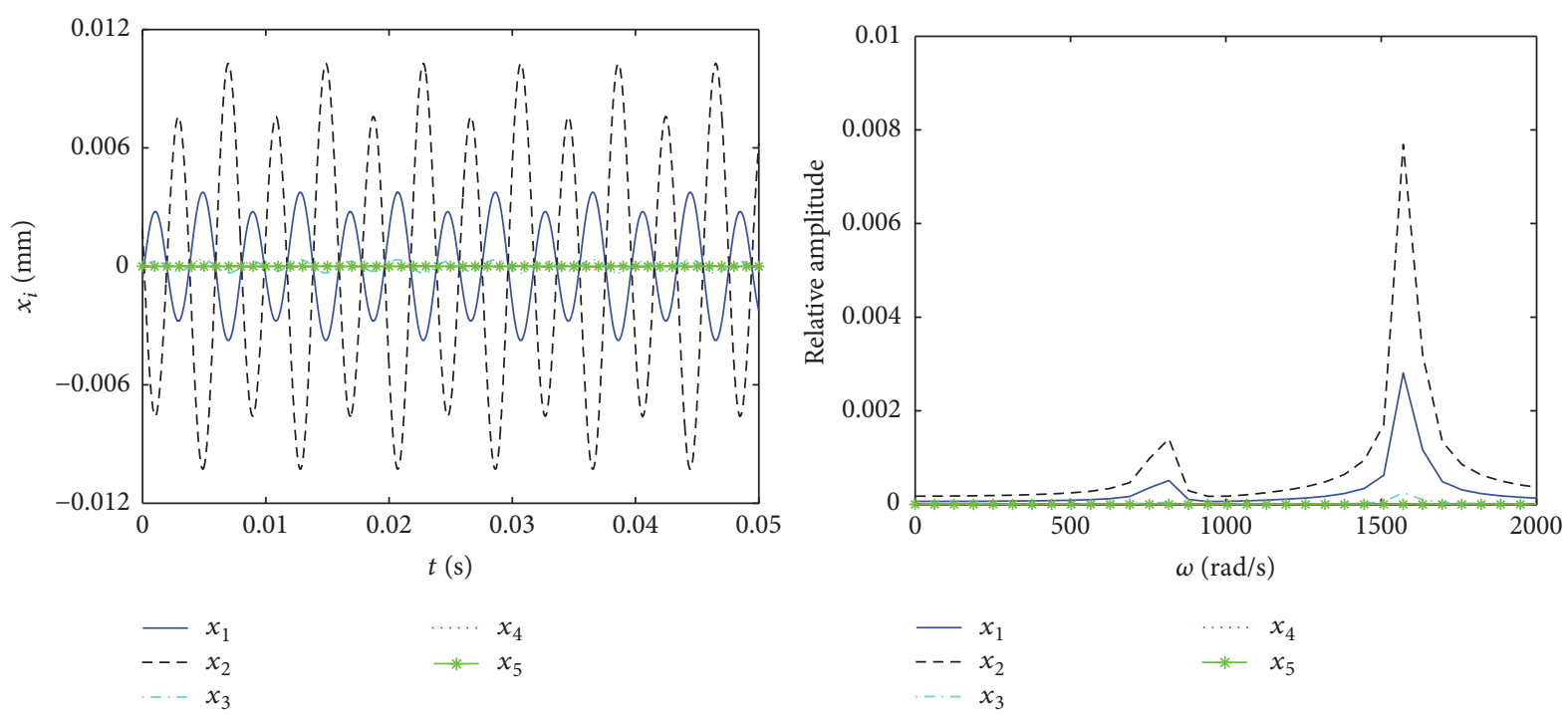

(b) $\omega_{e} \approx \omega_{2} / 2$
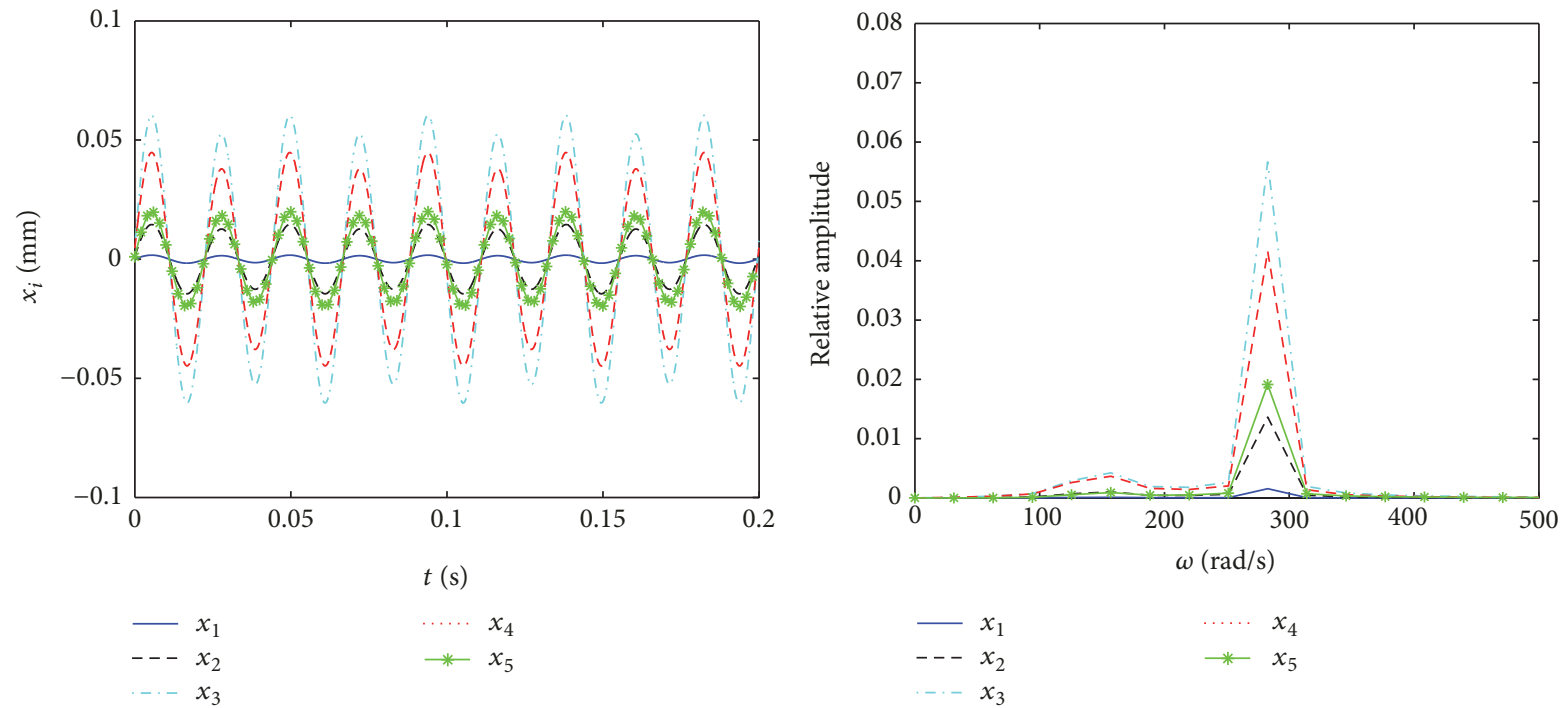

(c) $\omega_{e} \approx \omega_{3} / 2$

FIGURE 7: Continued. 

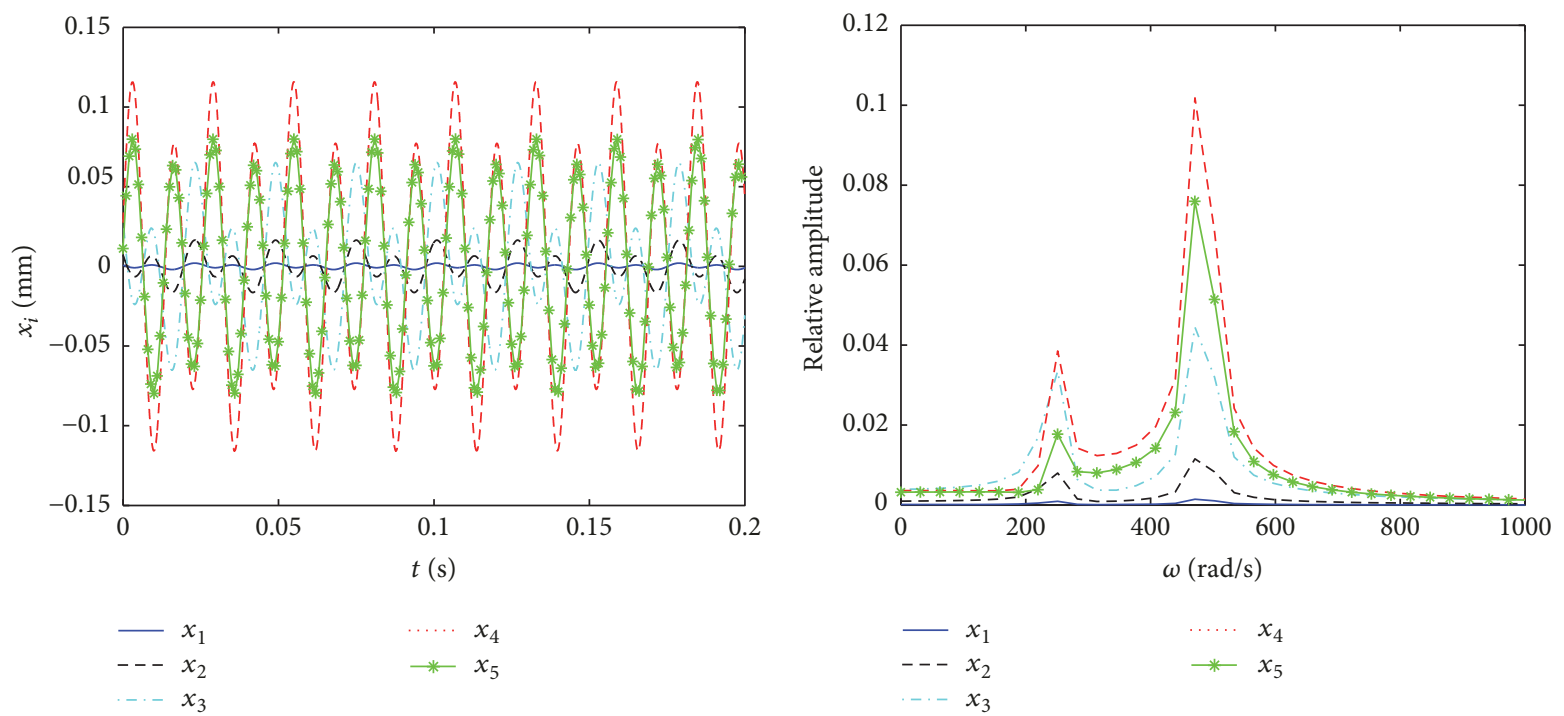

(d) $\omega_{e} \approx \omega_{4} / 2$
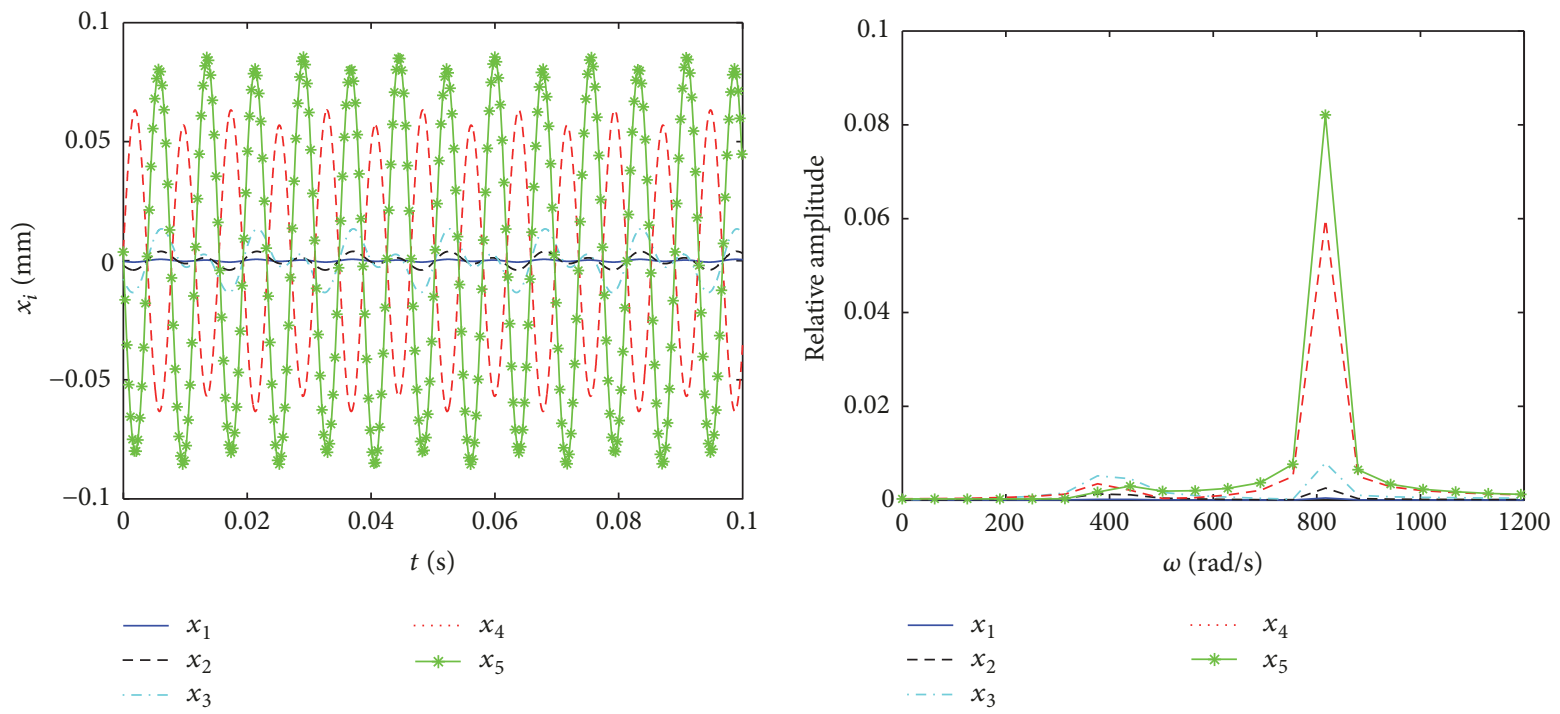

(e) $\omega_{e} \approx \omega_{5} / 2$

FIGURE 7: Main resonance when $\omega_{e} \approx \omega_{i} / 2$.

stator, will also be larger; however, the displacements of the interval components, namely, the inner FP and stator, will be much smaller.

Similarly, when the wave frequency is close to twice the natural frequency of the derived EIMG system, strong subharmonic resonance will occur because of the nonlinear magnetic coupling stiffness. The dynamic behavior is similar to the superharmonic response. When the outer stator outputs torque with a wave frequency close to the natural frequency of the derived EIMG system, strong resonance will occur. Furthermore, the amplitudes of the various components will be very different.

Because there is no contact among the components, the relative influences among adjacent (interval) components are larger (smaller) than those in a mechanical gear system. Because of the output torque on the outer FP waves, the equivalent load is greatest on the outer FP. Therefore, the largest resonance occurs when the wave frequency is close to the natural frequency of the outer FP rotational mode. Because the magnetic coupling stiffness is much smaller than the support stiffness, the natural frequencies of the inner rotor and outer FP rotational mode are much larger. When the current frequency is smaller, the EIMG can be used in applications that easily admit low frequencies, high torque, and low-frequency resonance. When the current frequency is larger, low-frequency main resonances may not occur; however, superharmonic resonances can easily occur.

\section{Conclusion}

EIMG systems output torque as a result of the magnetic coupling among components. The magnetic coupling stiffness is much smaller than the mesh stiffness of mechanical gear systems and exhibits distinct nonlinearity. Thus, there 
are quadratic nonlinearities in the EIMG dynamic system. When the wave frequency is close to the natural frequency or twice/half the natural frequency of the derived EIMG system, strong resonance will occur. The dominant frequency is the natural frequency, and the resonance amplitudes exhibit large differences when superharmonic resonance occurs. Because of the smaller magnetic coupling stiffness and damping, the transient responses slowly decay to zero. In order to accelerate the decay rate of the transient responses and avoid the resonances, the vibration control strategy of the EIMG system should be analyzed. These can provide the foundation for the parameter optimization and performance improvement of the EIMG system.

\section{Conflicts of Interest}

The authors declare that there are no conflicts of interest regarding the publication of this paper.

\section{Acknowledgments}

This project is supported by the National Natural Science Foundation of China (Grant no. 51675463) and the Natural Science Foundation of Hebei Province of China (E2017203098).

\section{References}

[1] S. Peng, W. N. Fu, and S. L. Ho, "A novel high torquedensity triple-permanent-magnet-excited magnetic gear," IEEE Transactions on Magnetics, vol. 50, no. 11, 2014.

[2] K. Atallah and D. Howe, "A novel high-performance magnetic gear," IEEE Transactions on Magnetics, vol. 37, no. 4, pp. 28442846, 2001.

[3] L. Baghli, E. Gouda, S. Mezani, and A. Rezzoug, "Hybrid vehicle with a magnetic planetary gear," Mediterranean Journal of Measurement and Control, vol. 7, no. 2, pp. 243-249, 2011.

[4] G. Bao, X. Liu, and K. Mao, "Characteristics of field modulated magnetic gear in wind turbine system," Transactions of the Chinese Society of Agricultural Machinery, vol. 42, no. 5, pp. 116150, 2011.

[5] N. W. Frank and H. A. Toliyat, "Gearing ratios of a magnetic gear for marine applications," in Proceedings of the IEEE Electric Ship Technologies Symposium (ESTS '09), pp. 477-481, Baltimore, Md, USA, April 2009.

[6] S. Mezani, K. Atallah, and D. Howe, "A high-performance axialfield magnetic gear," Journal of Applied Physics, vol. 99, no. 8, Article ID 08R303, 2006.

[7] K. Atallah, J. Wang, and D. Howe, "A high-performance linear magnetic gear," Journal of Applied Physics, vol. 97, no. 10, Article ID 10N516, 2005.

[8] Y. Liu, S. L. Ho, and W. N. Fu, "A novel magnetic gear with intersecting axes," IEEE Transactions on Magnetics, vol. 50, no. 11, 2014.

[9] C. C. Awah, Z. Q. Zhu, Z. Wu, and D. Wu, "High torque density magnetically-geared switched flux permanent magnet machines," in Proceedings of the 10th International Conference on Ecological Vehicles and Renewable Energies, EVER 2015, Monte Carlo, Monaco, April 2015.
[10] K. T. Chau, D. Zhang, J. Z. Jiang, C. Liu, and Y. Zhang, "Design of a magnetic-geared outer-rotor permanent-magnet brushless motor for electric vehicles," IEEE Transactions on Magnetics, vol. 43, no. 6, pp. 2504-2506, 2007.

[11] X.-H. Hao, H.-F. Zhang, and J.-D. Men, "Electromechanical integrated magnetic gear," Advances in Mechanical Engineering, vol. 8 , no. 6 , pp. 1-14, 2016.

[12] X. Hao, X. Zhu, and H. Zhang, "Free vibration of the electromechanical integrated magnetic gear system," Journal of Vibroengineering, vol. 17, no. 3, pp. 1120-1132, 2015.

[13] X.-H. Hao and X.-J. Zhu, "Forced responses of the parametric vibration system for the electromechanical integrated magnetic gear," Shock and Vibration, vol. 2015, Article ID 572937, 2015.

[14] N. W. Frank, S. Pakdelian, and H. A. Toliyat, "Passive suppression of transient oscillations in the concentric planetary magnetic gear," IEEE Transactions on Energy Conversion, vol. 26, no. 3, pp. 933-939, 2011.

[15] R. Montague, C. Bingham, and K. Atallah, "Servo control of magnetic gears," IEEE/ASME Transactions on Mechatronics, vol. 17, no. 2, pp. 269-278, 2012. 


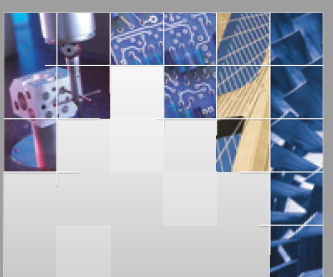

\section{Enfincering}
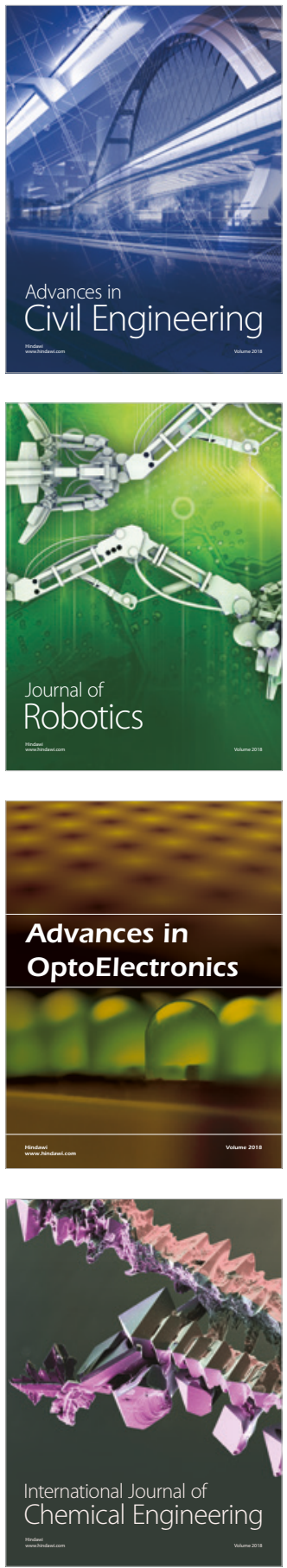

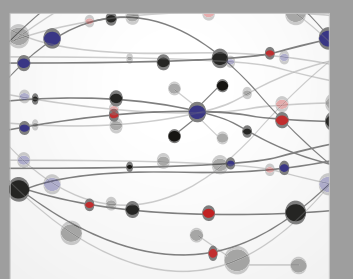

\section{Rotating \\ Machinery}

The Scientific World Journal

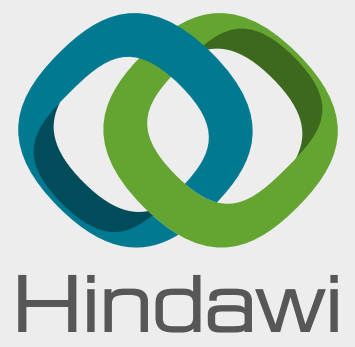

Submit your manuscripts at

www.hindawi.com
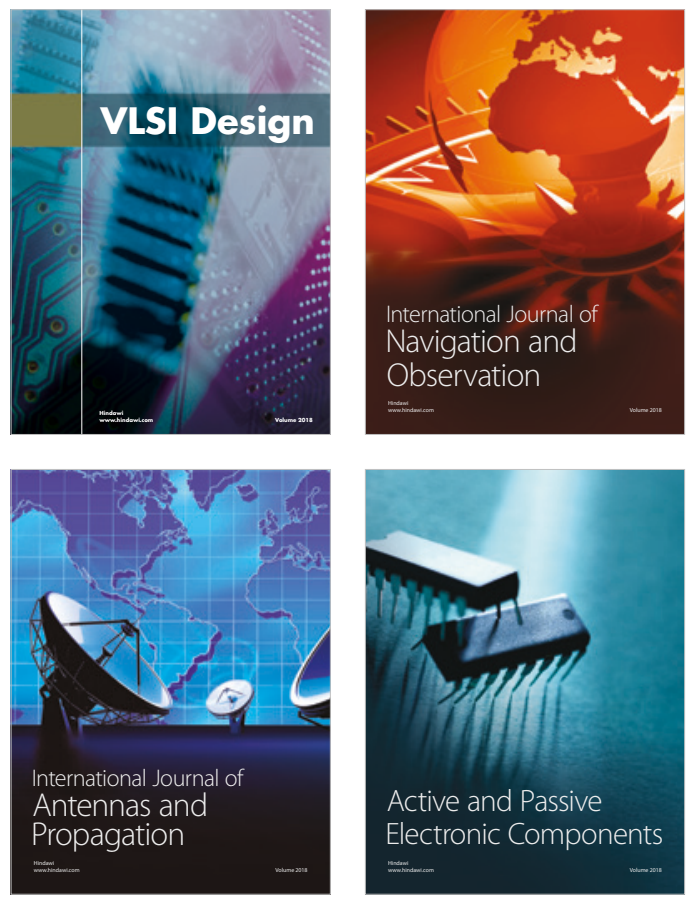
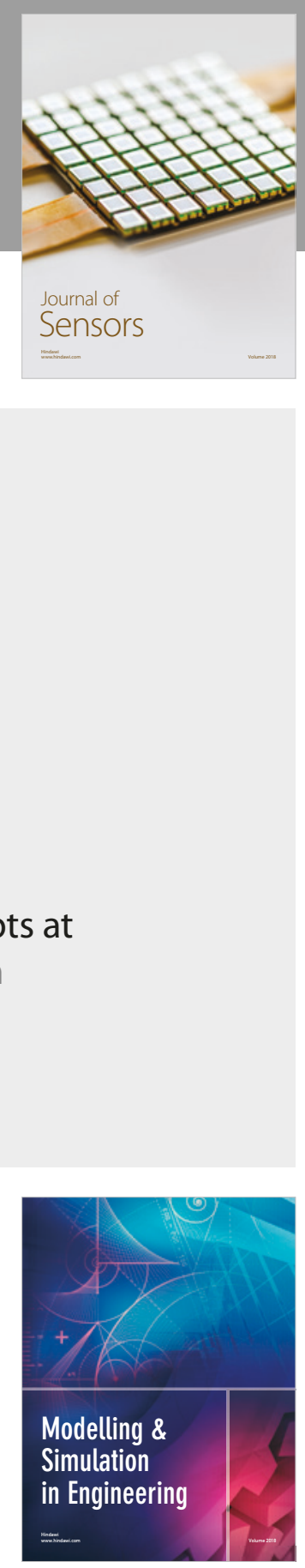

\section{Advances \\ Multimedia}
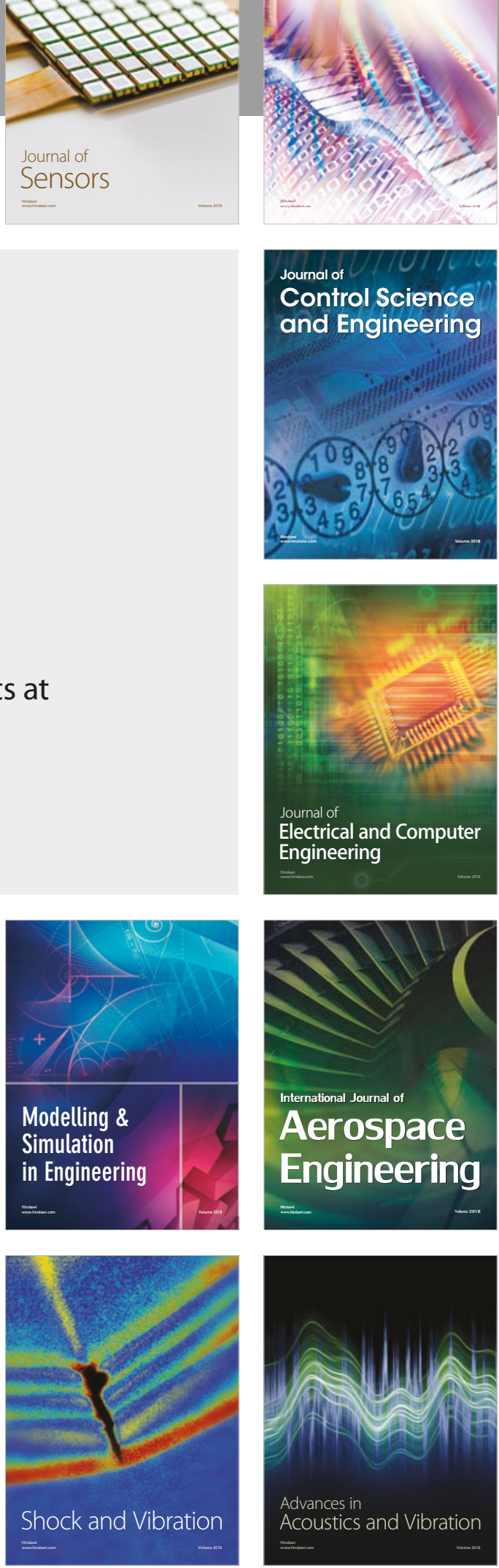